1 of 32

\title{
Finite element analysis for a diffusion equation on a harmonically evolving domain
}

\author{
DOMINIK EDELMANN $\dagger$ \\ Mathematisches Institut, Universität Tübingen \\ Auf der Morgenstelle 10, 72076 Tübingen, Germany
}

\begin{abstract}
We study convergence of the evolving finite element semi-discretization of a parabolic partial differential equation on an evolving bulk domain. The boundary of the domain evolves with a given velocity, which is then extended to the bulk by solving a Poisson equation. The numerical solution to the parabolic equation depends on the numerical evolution of the bulk, which yields the time-dependent mesh for the finite element method. The stability analysis works with the matrix-vector formulation of the semidiscretization only and does not require geometric arguments, which are then required in the proof of consistency estimates. We present various numerical experiments that illustrate the proven convergence rates.
\end{abstract}

Keywords: evolving domain, harmonic velocity, diffusion, evolving finite elements, error analysis

Classification...

\section{Introduction}

This paper studies the numerical discretization of a diffusion equation in a time-dependent domain that is specified by the velocity of its boundary. The interior velocity is determined as the solution of a Laplace equation with the given boundary velocity as Dirichlet data.

The strong formulation of this model is to find the time-dependent domain $\Omega(t) \subset \mathbb{R}^{n}(n=2,3)$, $t \in[0, T]$, which moves with a velocity $v$ that is the harmonic extension of the a priori given velocity $v^{\Gamma}$ of the boundary $\Gamma(t)=\partial \Omega(t)$. That is, $v$ is not given explicitly but determined as the solution of the Laplace equation, for all $t \in[0, T]$,

$$
\begin{aligned}
-\Delta v(x, t) & =0, & & x \in \Omega(t), \\
v(x, t) & =v^{\Gamma}(x, t), & & x \in \Gamma(t) .
\end{aligned}
$$

In $\Omega(t)$ we seek a solution $u=u(x, t)$ with given initial data $u(\cdot, 0)=u_{0}$ to the partial differential equation

$$
\partial^{\bullet} u(x, t)+u(x, t) \nabla \cdot v(x, t)-\beta \Delta u(x, t)=f(x, t), \quad x \in \Omega(t), \quad t \in[0, T],
$$

where $\partial^{\bullet}$ denotes the material derivative, $\nabla \cdot v$ is the divergence of the velocity and $\beta>0$ is a given diffusion coefficient. On the boundary, we impose the Neumann condition $\frac{\partial u}{\partial \mathrm{n}}(x, t)=g(x, t), x \in \Gamma(t)$, $t \in[0, T]$, where $\mathrm{n}$ denotes the unit outward pointing normal to $\Gamma(t)$.

Convection-diffusion equations in time-dependent domains have gained considerable interest in the past decades. A model similar to (1.2) (without (1.1)) with an additional convection term, together with homogeneous Dirichlet boundary conditions is analyzed in Badia \& Codina (2006) and Boffi \&

${ }^{\dagger}$ Email: edelmann@na.uni-tuebingen.de 
Gastaldi (2004) in an arbitrary Lagrangian-Eulerian (ALE) framework, where the velocity is determined by the ALE mapping. In Boffi \& Gastaldi (2004), the velocity of the boundary is prescribed and the ALE mapping is constructed as the harmonic extension of the boundary positions. This approach is first proposed in Formaggia \& Nobile (1999) in the context of a generic conservation law on a moving domain, see also Gastaldi (2001); Formaggia \& Nobile (2004) and the references therein.

Diffusion equations on evolving surfaces are analyzed in Dziuk \& Elliott (2007a,b, 2013) for a given velocity, and there are recent works, where the velocity is not given explicitly but determined by various velocity laws that depend on the solution of the diffusion equation on the surface, see Kovács et al. (2017); Kovács \& Lubich (2018); Kovács et al. (2019a).

Through the numerical analysis of the problem with a given boundary velocity (1.1)-(1.2) we will develop techniques which are expected to be essential for more involved problems, such as the tumor growth model of Eyles et al. (2019), where a bulk-surface model for tissue growth is presented, together with a numerical algorithm. Instead of the coupled system (1.1)-(1.2) in (Eyles et al., 2019, (1.1)-(1.3) $\&$ Section 6.1.2) they consider the boundary velocity $v^{\Gamma}$ given by the forced mean curvature flow

$$
v^{\Gamma}=\frac{u}{\alpha}+\beta H
$$

and instead of (1.2) they consider an elliptic boundary value problem in the moving bulk. Here $H$ denotes the mean curvature of the boundary surface and $\alpha, \beta$ are given positive constants.

In this paper, we prove error bounds for the spatial semi-discretization of the coupled problem (1.1)(1.2) with isoparametric finite elements of polynomial degree at least two. More precisely, we show $H^{1}$-norm error bounds in the positions and the velocity $v$ that are uniform in time, and $L^{\infty} L^{2}$-norm and $L^{2} H^{1}$-norm error bounds for the solution $u$ of the diffusion equation. The proof clearly separates the stability and consistency analysis. To prove stability of the semi-discrete equations, we adapt techniques recently used in Kovács et al. (2017); Kovács (2017) to the present situation. The stability analysis of the semi-discrete problems uses energy estimates. Transport formulae are used to relate mass and stiffness matrices corresponding to different discrete domains. In order to estimate errors between these matrices on different domains, a key issue is to control the $W^{1, \infty}$-norm of the position error uniformly in time. This is done with an inverse estimate, that yields an $\mathscr{O}\left(h^{k-n / 2}\right)$ bound uniformly in time, which is small only for $k \geqslant 2$. The stability analysis of the semi-discrete diffusion equation uses the same techniques and is based on the stability analysis of the semi-discrete velocity law. Moreover, it becomes clear how the position error affects the error in the numerical solution to (1.2).

The stability analysis relies on smallness assumptions on the defects. These are shown to be true in the following consistency analysis, that uses geometric approximation estimates and interpolation results. The final convergence result is then obtained by combining stability and consistency estimates together with interpolation error bounds.

The paper is organized as follows.

In Section 2 we recall basic notation and formulate a diffusion equation on an evolving domain together with the above velocity law. We derive the weak formulation.

In Section 3 we describe the high-order evolving finite element approximation of the problem. After introducing an exact triangulation of the curved domain, we define the computational domain and the finite element method. We describe the spatial semi-discretization and derive a matrix-vector formulation, which will be crucial for the stability analysis.

In Section 4 we state the main result of the paper, which gives convergence estimates for the spatial semi-discretization with evolving isoparametric finite elements of polynomial degree at least 2 . We outline the main ideas of the proof. 
In Section 5 we collect auxiliary results that will be needed for the following analysis. The first part deals with the evolving mass and stiffness matrices and their properties, which are crucial in the stability analysis. The second part collects geometric estimates which will be needed only for consistency analysis.

Section 6 analyses the stability of the semi-discrete velocity law without a diffusion equation on the evolving domain. In Section 7, we extend the stability analysis to the semi-discrete diffusion equation. Section 8 contains the consistency analysis, that is, estimating the defects obtained on inserting the interpolated exact solutions into the numerical scheme.

In Section 9 we prove the main convergence result by combining the stability and consistency estimates. Section 10 provides several numerical experiments which illustrate the theoretical results.

\section{Problem formulation}

\subsection{Basic notation}

For $t \in[0, T]$, let $\Omega(t) \subseteq \mathbb{R}^{n}(n=2,3)$ be an open, bounded and connected set with smooth boundary $\Gamma(t)=\partial \Omega(t)$ and $\Omega_{0}=\Omega(0), \Gamma_{0}=\Gamma(0)$. We denote $\overline{\Omega(t)}=\Omega(t) \cup \Gamma(t)$. We assume that there exists a sufficiently smooth map $X: \Omega_{0} \cup \Gamma_{0} \times[0, T] \rightarrow \mathbb{R}^{n}$ such that

$$
\Omega(t)=\left\{X(p, t): p \in \Omega_{0}\right\}, \quad \Gamma(t)=\left\{X(p, t): p \in \Gamma_{0}\right\} .
$$

The velocity $v(x, t)$ at a point $x=X(p, t) \in \overline{\Omega(t)}$ is defined by

$$
v(X(p, t), t)=\frac{\partial}{\partial t} X(p, t) .
$$

For a function $u=u(x, t), x \in \overline{\Omega(t)}, t \in[0, T]$, the material derivative at $x=X(p, t)$ is defined by

$$
\partial^{\bullet} u(x, t)=\frac{\mathrm{d}}{\mathrm{d} t} u(X(p, t), t)=\frac{\partial}{\partial t} u(x, t)+\nabla u(x, t) \cdot v(x, t) .
$$

For $x \in \Gamma(t)$, we denote by $\mathrm{n}=\mathrm{n}(x, t)$ the unit outward pointing normal to $\Gamma(t)$. We define the spacetime domain $\Omega_{T}$ and the space-time surface $\Gamma_{T}$ by

$$
\Omega_{T}=\bigcup_{t \in[0, T]} \Omega(t) \times\{t\}, \quad \Gamma_{T}=\bigcup_{t \in[0, T]} \Gamma(t) \times\{t\} .
$$

For functions $\varphi, \psi$ defined on $\Omega(t)$, we have bilinear forms

$$
\begin{aligned}
& m(\varphi, \psi)=\int_{\Omega(t)} \varphi \psi \mathrm{d} x, \\
& a(\varphi, \psi)=\int_{\Omega(t)} \nabla \varphi \cdot \nabla \psi \mathrm{d} x .
\end{aligned}
$$

Note that these bilinear forms explicitly depend on $t$, but we will omit the argument $t$, for brevity. It will always be clear from context for which $t \in[0, T]$ the bilinear forms are evaluated. 


\subsection{Diffusion equation}

We assume that $u=u(\cdot, t)$ is the density of a scalar quantity on $\Omega(t)$ (for example, mass per unit volume). We follow a construction of (Dziuk \& Elliott, 2007a, Section 3 ) to obtain a diffusion equation with Neumann boundary conditions:

$$
\left\{\begin{aligned}
\partial^{\bullet} u+u \nabla \cdot v-\beta \Delta u=f & \text { in } \quad \\
\frac{\partial u}{\partial \mathrm{n}}=\nabla u \cdot \mathrm{n}=g & \text { on } \quad \Gamma(t),
\end{aligned}\right.
$$

where $\nabla \cdot v$ denotes the divergence of the velocity, $\beta>0$ is a given diffusion coefficient and $\mathrm{n}$ the unit outward pointing normal to $\Gamma(t)$.

\subsection{Harmonic velocity law}

Contrary to existing works (cf. Elliott \& Ranner (2017)), the velocity $v(\cdot, t)$ of $\Omega(t)$ is not given explicitly. Instead, only the velocity of the boundary $\Gamma(t)=\partial \Omega(t)$ is given; the velocity of the bulk is then determined as the harmonic extension, i.e. as the solution to the Laplace equation. More precisely, we have the following differential equation for $v(x, t)$ : for each $t \in[0, T]$

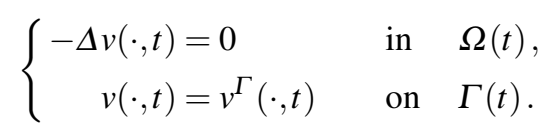

We assume that $v^{\Gamma}$ is defined on a neighborhood of $\Gamma_{T}$, as defined in (2.2). This system is considered together with the position ODEs: for each $p \in \overline{\Omega(0)}$

$$
\left\{\begin{aligned}
\frac{\mathrm{d}}{\mathrm{d} t} X(p, t) & =v(X(p, t), t), \\
X(p, 0) & =p .
\end{aligned}\right.
$$

We consider an equivalent problem with homogeneous Dirichlet boundary conditions: assume that $v^{\Gamma}(\cdot, t)$ is the trace of a given function $w(\cdot, t) \in H^{1}(\Omega(t))^{n}$ and consider the equivalent problem: find $\widetilde{v}(\cdot, t) \in H_{0}^{1}(\Omega(t))^{n}$ such that

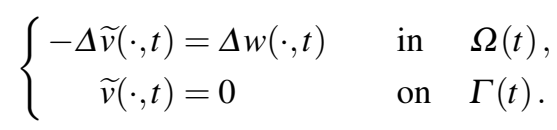

It is easily seen that the solution $v=\widetilde{v}+w$ to (2.5) does not depend on the choice of $w$.

\subsection{Coupled problem: strong and weak formulation}

We consider the following system of partial differential equations: for given $\beta>0, f: \mathbb{R}^{n} \times[0, T] \rightarrow \mathbb{R}$, $g: \mathbb{R}^{n} \times[0, T] \rightarrow \mathbb{R}$ and $v^{\Gamma}: \mathbb{R}^{n} \times[0, T] \rightarrow \mathbb{R}^{n}$, find the unknown function $u: \Omega_{T} \rightarrow \mathbb{R}$, the unknown velocity field $v: \Omega_{T} \rightarrow \mathbb{R}^{n}$ and the unknown position function $X: \Omega_{0} \cup \Gamma_{0} \times[0, T] \rightarrow \mathbb{R}^{n}$ such that for 
all $t \in[0, T]$

$$
\left\{\begin{aligned}
\partial \bullet u(\cdot, t)+u(\cdot, t) \nabla \cdot v(\cdot, t)-\beta \Delta u(\cdot, t) & =f(\cdot, t) & & \text { in } \quad \Omega(t), \\
\frac{\partial u}{\partial \mathrm{n}}(\cdot, t) & =g(\cdot, t) & & \text { on } \quad \Gamma(t), \\
\frac{\mathrm{d} X}{\mathrm{~d} t}(\cdot, t) & =v(X(\cdot, t), t) & & \text { in } \quad \Omega_{0} \cup \Gamma_{0}, \\
-\Delta v(\cdot, t) & =0 & & \text { in } \quad \Omega(t), \\
v(\cdot, t) & =v^{\Gamma}(\cdot, t) & & \text { on } \quad \Gamma(t) .
\end{aligned}\right.
$$

Without loss of generality, we assume $\beta=1$ and $g \equiv 0$ in the following.

REMARK 2.1 The last three equations of (2.7) purely describe the motion of the domain $\Omega(t)$ and are independent of the parabolic equation for $u$. The latter includes the velocity $v$ in the material derivative as well as the divergence of the velocity in the equation. This is reflected in the stability analysis, which is first done for the discretization of the domain motion, and then extended to the parabolic equation. On the other hand, if the velocity field $v$ was given for the whole domain, the finite element analysis for the parabolic equation alone would be remarkably easier. Convergence results for these types of problems with given velocity on the whole domain are found in (Elliott \& Ranner, 2017, Section 7).

We now derive a weak formulation. By multiplying the first equation with an arbitrary test function $\varphi \in H^{1}(\Omega(t))$ such that $\partial^{\bullet} \varphi$ exists in $L^{2}(\Omega(t))$, integrating over $\Omega(t)$, using the Leibniz formula, Green's formula and the Neumann boundary condition, we arrive at

$$
\frac{\mathrm{d}}{\mathrm{d} t} \int_{\Omega(t)} u \varphi+\int_{\Omega(t)} \nabla u \cdot \nabla \varphi=\int_{\Omega(t)} f \varphi+\int_{\Omega(t)} u \partial^{\bullet} \varphi .
$$

Multiplying (2.6) with arbitrary test function $\psi \in H_{0}^{1}(\Omega(t))^{n}$, integrating over $\Omega(t)$ and using Green's formula, we obtain

$$
\int_{\Omega(t)} \nabla \widetilde{v} \cdot \nabla \psi=-\int_{\Omega(t)} \nabla w \cdot \nabla \varphi
$$

where we have used that the boundary integrals vanish thanks to $\psi \in H_{0}^{1}(\Omega(t))^{n}$. Here, the dot denotes the Euclidean inner product of the vectorizations of the matrices, i.e. the Frobenius norm inner product of the matrices. Again, it can be shown that the weak solution $v=\widetilde{v}+w$ does not depend on $w$.

The weak formulation of the diffusion equation and the domain evolution thus reads: find $u(\cdot, t) \in$ $H^{1}(\Omega(t)), \widetilde{v}(\cdot, t) \in H_{0}^{1}(\Omega(t))^{n}$ such that for all $\varphi \in H^{1}(\Omega(t))$ with $\partial^{\bullet} \varphi \in L^{2}(\Omega(t)), \psi \in H_{0}^{1}(\Omega(t))^{n}$ and all $t \in[0, T]$

$$
\begin{aligned}
\frac{\mathrm{d}}{\mathrm{d} t} \int_{\Omega(t)} u \varphi+\int_{\Omega(t)} \nabla u \cdot \nabla \varphi & =\int_{\Omega(t)} f \varphi+\int_{\Omega(t)} u \partial^{\bullet} \varphi \\
\int_{\Omega(t)} \nabla \widetilde{v} \cdot \nabla \psi & =-\int_{\Omega(t)} \nabla w \cdot \nabla \psi \\
v & =\widetilde{v}+w, \\
\frac{\mathrm{d} X}{\mathrm{~d} t} & =v .
\end{aligned}
$$


This is considered together with given initial data $u(\cdot, t)=u_{0}(\cdot), X(\cdot, 0)=I d$.

We assume throughout the paper that there exists a unique weak solution with sufficiently high Sobolev regularity on $[0, T]$. Precise regularity assumptions will be given in Theorem 4.1.

From now on, we will be working in the technically more challenging three-dimensional case. All of the upcoming results are valid in the two-dimensional case as well.

\section{Evolving bulk finite elements}

In this section we briefly recall the evolving isoparametric finite element method which is used for semidiscretization in space. We refer to Elliott \& Ranner $(2013,2017)$ for a more detailed introduction into the construction of isoparametric finite elements .

In the following, we denote $\Omega_{0}=\Omega(0)$ for brevity. The initial domain $\Omega_{0}$ is triangulated and the nodes are then evolved in time by solving the position ODE $\dot{x}_{i}=v\left(x_{i}, t\right)$ in each node, together with (2.5).

\subsection{High-order domain approximation}

We construct a triangulation $\mathscr{T}_{h}^{(1)}$ of $\Omega_{0}$ consisting of closed simplices with maximal diameter $h$. The union of all simplices of $\mathscr{T}_{h}^{(1)}$ defines a polyhedral approximation $\Omega_{h}$ of $\Omega_{0}$, whose boundary $\Gamma_{h}=\partial \Omega_{h}$ is an interpolation of $\Gamma_{0}$.

Each simplex $T \in \mathscr{T}_{h}^{(1)}$ corresponds to a curved simplex $T^{\mathrm{c}} \subset \Omega$, which is parametrized over the unit simplex $\widehat{T}$ with a map $\Phi_{T}^{\mathrm{c}}=\Phi_{T}+\rho_{T}$. Here, $\Phi_{T}$ denotes the usual affine function that maps $\widehat{T}$ onto $T$. For the construction of an appropriate $\rho_{T}$, we refer to Elliott $\&$ Ranner (2013). The union of those curved simplices can be considered as an exact triangulation of $\Omega_{0}$. Using the map $\Phi_{T}^{\mathrm{c}}$, we can define an isoparametric mapping $\Phi_{T}^{(k)}$, that maps the unit simplex $\widehat{T}$ to a polynomial simplex $T^{(k)} . \Omega_{h}^{(k)}$ is then defined as the union of elements in $\mathscr{T}_{h}^{(k)}$, where

$$
\mathscr{T}_{h}^{(k)}:=\left\{T^{(k)}: T \in \mathscr{T}_{h}^{(1)}\right\}, \quad T^{(k)}:=\left\{\Phi_{T}^{(k)}(\widehat{x}): \widehat{x} \in \widehat{T}\right\} .
$$

\subsection{Evolving finite element method}

Here and in the following, we use the notational convention that vectors and matrices are denoted with bold-face letters. As mentioned, we set $n=3$ and assume that the order $k \geqslant 2$ is fixed.

Based on the previous subsection, we obtain a triangulation of $\Omega_{0}$, whose nodes $x_{1}^{0}, \ldots, x_{N}^{0}$ are collected in a vector $\mathbf{x}^{0}=\left(x_{1}^{0}, \ldots, x_{N}^{0}\right) \in \mathbb{R}^{3 N}$. We assume that the enumeration is such that exactly the first $N_{\Gamma}$ nodes lie on the boundary $\Gamma_{0}=\partial \Omega_{0}$. The nodes are evolved in time and collected in a vector $\mathbf{x}(t)=\left(x_{1}(t), \ldots, x_{N}(t)\right)$ with $\mathbf{x}(0)=\mathbf{x}^{0}$. We use the notation

$$
\mathbf{x}(t)=\left(\begin{array}{c}
\mathbf{x}^{\Gamma}(t) \\
\mathbf{x}^{\Omega}(t)
\end{array}\right)
$$

to indicate which nodes live on the boundary. The nodal vector $\mathbf{x}=\mathbf{x}(t)$ defines a computational domain $\Omega_{h}(\mathbf{x})=\Omega_{h}(\mathbf{x}(t))$ with boundary $\Gamma_{h}(\mathbf{x})$. The finite element basis functions $\varphi_{j}(\cdot, t): \Omega_{h}(\mathbf{x}(t)) \rightarrow \mathbb{R}$ satisfy

$$
\varphi_{j}\left(x_{k}(t), t\right)=\delta_{j k}, \quad 1 \leqslant j, k \leqslant N
$$


and their pullback to the reference triangle is polynomial of degree $k$. Note that, since the velocity is not given explicitly, we are in general not able to find the exact positions $x_{j}^{*}(t)=X\left(x_{j}^{0}, t\right)$, so that $\Omega_{h}(\mathbf{x}(t))$ is not the triangulation of $\Omega(t)$ corresponding to the exact positions $X\left(x_{j}^{0}, t\right)$. It is therefore more convenient to denote the dependence on $\mathbf{x}$ instead of $t$, i.e. to write $\Omega_{h}(\mathbf{x})$ and not $\Omega_{h}(t)$, etc.

The finite element space is now given as

$$
S_{h}(\mathbf{x})=\operatorname{span}\left\{\varphi_{1}[\mathbf{x}], \ldots, \varphi_{N}[\mathbf{x}]\right\},
$$

where $\varphi_{j}[\mathbf{x}](\cdot)=\varphi_{j}(\cdot, t)$ for $\mathbf{x}=\mathbf{x}(t)$.

We use the notation

$$
S_{0, h}(\mathbf{x})=\left\{\varphi_{h}[\mathbf{x}] \in S_{h}(\mathbf{x}): \gamma_{h} \varphi_{h}[\mathbf{x}]=0\right\}=\operatorname{span}\left\{\varphi_{N_{\Gamma}+1}(\mathbf{x}), \ldots, \varphi_{N}[\mathbf{x}]\right\},
$$

where $\gamma_{h} \varphi_{h}$ denotes the trace of a function $\varphi_{h}$ defined on $\Omega_{h}(\mathbf{x})$ on $\Gamma_{h}(\mathbf{x})$. We set

$$
X_{h}\left(p_{h}, t\right)=\sum_{j=1}^{N} x_{j}(t) \varphi_{j}[\mathbf{x}(0)]\left(p_{h}\right), \quad p_{h} \in \Omega_{h}^{0} \cup \Gamma_{h}^{0},
$$

which has the properties that $X_{h}\left(x_{k}^{0}, t\right)=x_{k}(t)$ and $X_{h}\left(x_{j}^{0}, 0\right)=x_{j}^{0}$, implying that $X_{h}(x, 0)=x$ for all $x \in \Omega_{h}^{0} \cup \Gamma_{h}^{0}$. The discrete velocity $v_{h}(x, t)$ at a particle $x=X_{h}\left(p_{h}, t\right)$ is given by

$$
v_{h}\left(X_{h}\left(p_{h}, t\right), t\right)=\frac{\mathrm{d}}{\mathrm{d} t} X_{h}\left(p_{h}, t\right) .
$$

The basis functions satisfy the transport property

$$
\frac{\mathrm{d}}{\mathrm{d} t}\left(\varphi_{j}[\mathbf{x}(t)]\left(X_{h}\left(p_{h}, t\right)\right)\right)=0,
$$

which implies $\varphi_{j}[\mathbf{x}(t)]\left(X_{h}\left(p_{h}, t\right)\right)=\varphi_{j}[\mathbf{x}(0)]\left(p_{h}\right)$. For the discrete velocity, this means

$$
v_{h}(x, t)=v_{h}\left(X_{h}\left(p_{h}, t\right), t\right)=\frac{\mathrm{d}}{\mathrm{d} t} \sum_{j=1}^{N} x_{j}(t) \varphi_{j}[\mathbf{x}(0)]\left(p_{h}\right)=\sum_{j=1}^{N} v_{j}(t) \varphi_{j}[\mathbf{x}(t)](x) \text { with } v_{j}=\dot{x}_{j} .
$$

In particular, $v_{h}(\cdot, t) \in S_{h}(\mathbf{x}(t))$. For a finite element function $u_{h}(x, t)=\sum_{j=1}^{N} u_{j}(t) \varphi_{j}[\mathbf{x}(t)](x)$, the discrete material derivative at $x=X_{h}\left(p_{h}, t\right)$ is defined by

$$
\partial_{h}^{\bullet} u_{h}(x, t)=\frac{\mathrm{d}}{\mathrm{d} t} u_{h}\left(X_{h}\left(p_{h}, t\right), t\right)=\sum_{j=1}^{N} \dot{u}_{j}(t) \varphi_{j}[\mathbf{x}(t)](x),
$$

where we have used the transport property again. In particular: $\partial_{h}^{\bullet} u_{h}(\cdot, t) \in S_{h}(\mathbf{x}(t))$.

\subsection{Spatial semi-discretization and matrix-vector formulation}

The evolving finite element discretization of (2.8) reads: find the unknown position vector $\mathbf{x}(t) \in \mathbb{R}^{3 N}$ and the unknown finite element functions $u_{h}(\cdot, t) \in S_{h}(\mathbf{x}(t)), \widetilde{v}_{h}(\cdot, t) \in S_{0, h}(\mathbf{x}(t))^{3}$ such that for all $\varphi_{h}(\cdot, t) \in S_{h}(\mathbf{x}(t))$ with $\partial_{h}^{\bullet} \varphi_{h} \in S_{h}(\mathbf{x}(t))$ and all $\psi_{h}(\cdot, t) \in S_{0, h}(\mathbf{x}(t))^{3}$

$$
\begin{gathered}
\frac{\mathrm{d}}{\mathrm{d} t} \int_{\Omega_{h}(\mathbf{x}(t))} u_{h} \varphi_{h}+\int_{\Omega_{h}(\mathbf{x}(t))} \nabla u_{h} \cdot \nabla \varphi_{h}=\int_{\Omega_{h}(\mathbf{x}(t))} f \varphi_{h}+\int_{\Omega_{h}(\mathbf{x}(t))} u_{h} \partial_{h}^{\bullet} \varphi_{h}, \\
\int_{\Omega_{h}(\mathbf{x}(t))} \nabla \widetilde{v}_{h} \cdot \nabla \psi_{h}=-\int_{\Omega_{h}(\mathbf{x}(t))} \nabla w_{h} \cdot \nabla \psi_{h},
\end{gathered}
$$


together with

$$
\frac{\partial}{\partial t} X_{h}\left(p_{h}, t\right)=v_{h}\left(X_{h}\left(p_{h}, t\right), t\right), \quad X_{h}\left(p_{h}, 0\right)=p_{h},
$$

for $p_{h} \in \Omega_{h}^{0} \cup \Gamma_{h}^{0}$, where $v_{h}=\widetilde{v}_{h}+w_{h}$. The initial values for the nodal vector $\mathbf{u}$ corresponding to $u_{h}(\cdot, 0)$ and the nodal vector $\mathbf{x}(0)$ are taken as the exact initial values of the nodes $x_{j}^{0}$ of the initial triangulation of $\Omega_{0}$ :

$$
u_{j}(0)=u\left(x_{j}^{0}, 0\right), \quad x_{j}(0)=x_{j}^{0} \quad(j=1, \ldots, N) .
$$

We now show that the nodal vectors $\mathbf{u} \in \mathbb{R}^{N}$ and $\mathbf{v} \in \mathbb{R}^{3 N}$ corresponding to the finite element functions $u_{h}$ and $v_{h}$, respectively, together with the position vector $\mathbf{x} \in \mathbb{R}^{3 N}$ satisfy a system of differential equations. We set (omitting the omnipresent $\operatorname{argument} t$ )

$$
u_{h}=\sum_{j=1}^{N} u_{j} \varphi_{j}[\mathbf{x}], \quad v_{h}=\sum_{j=1}^{N} v_{j} \varphi_{j}[\mathbf{x}]
$$

with $u_{j} \in \mathbb{R}, v_{j} \in \mathbb{R}^{3}$ and collect the nodal values in vectors $\mathbf{u} \in \mathbb{R}^{N}, \mathbf{v} \in \mathbb{R}^{3 N}$. The domain-dependent mass and stiffness matrices $\mathbf{M}(\mathbf{x})$ and $\mathbf{A}(\mathbf{x})$ are defined by

$$
\begin{aligned}
\mathbf{M}(\mathbf{x})_{j k} & =\int_{\Omega_{h}(\mathbf{x})} \varphi_{j}[\mathbf{x}] \varphi_{k}[\mathbf{x}] \mathrm{d} x, \\
\mathbf{A}(\mathbf{x})_{j k} & =\int_{\Omega_{h}(\mathbf{x})} \nabla \varphi_{j}[\mathbf{x}] \cdot \nabla \varphi_{k}[\mathbf{x}] \mathrm{d} x .
\end{aligned}
$$

In view of the following discretization of the velocity law, we use the notation

$$
\mathbf{A}(\mathbf{x})=\left(\begin{array}{ll}
\mathbf{A}_{11}(\mathbf{x}) & \mathbf{A}_{12}(\mathbf{x}) \\
\mathbf{A}_{21}(\mathbf{x}) & \mathbf{A}_{22}(\mathbf{x})
\end{array}\right)
$$

where $\mathbf{A}_{11}(x) \in \mathbb{R}^{N_{\Gamma} \times N_{\Gamma}}$ and $\mathbf{A}_{22}(\mathbf{x}) \in \mathbb{R}^{N_{\Omega} \times N_{\Omega}}$. $\mathbf{A}_{22}(\mathbf{x})$ thus corresponds to the finite element functions which vanish on the boundary. We will use the same notation for $\mathbf{M}(\mathbf{x})$ when it is necessary. It is important to note that the sub-matrix $\mathbf{A}_{22}(\mathbf{x})$ is invertible.

For the right-hand side of the diffusion equation, we define the vector

$$
\mathbf{f}(\mathbf{x}(t))_{k}=\int_{\Omega_{h}(\mathbf{x})} f \varphi_{k}[\mathbf{x}] \mathrm{d} x .
$$

By linearity, the transport property implies $\partial_{h}^{\bullet} \varphi_{h}=0$, so the first equation of (3.2) is equivalent to

$$
\frac{\mathrm{d}}{\mathrm{d} t}(\mathbf{M}(\mathbf{x}(t)) \mathbf{u}(t))+\mathbf{A}(\mathbf{x}(t)) \mathbf{u}(t)=\mathbf{f}(\mathbf{x}(t)) .
$$

For the velocity law, we remind that the nodes $x_{j}(t), j=1, \ldots, N_{\Gamma}$, on the boundary are known explicitly since $v^{\Gamma}(\cdot, t)$ is prescribed. Writing $v_{j}(t)=v^{\Gamma}\left(x_{j}(t), t\right)$, we have the finite element interpolation of $v^{\Gamma}$ :

$$
v_{h}^{\Gamma}(\cdot, t)=\sum_{j=1}^{N_{\Gamma}} v_{j}(t) \varphi_{j}[\mathbf{x}(t)](\cdot) .
$$


We write

$$
w_{h}(\cdot, t)=\sum_{j=1}^{N} w_{j}(t) \varphi_{j}[\mathbf{x}(t)](\cdot), \quad w_{j}(t)=v_{j}(t) \text { for } j=1, \ldots, N_{\Gamma},
$$

for an arbitrary extension of $v_{h}^{\Gamma}$. Noting that $\mathbf{v}$ has three components, a short calculation shows that the second equation of (3.2) is equivalent to

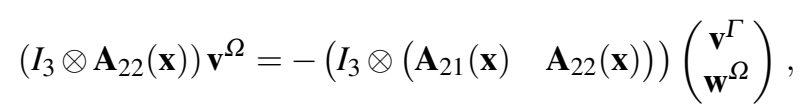

where $\mathbf{w}^{\Omega}$ is the vector containing the nodal values of $w_{h}$ in the inner nodes. Here, $I_{3}$ denotes the identity matrix of size $3 \times 3$ and $\otimes$ denotes the Kronecker product. The solution $v_{h}$ we are seeking is then obtained by $v_{h}=v_{h}^{\Gamma}+\widetilde{v}_{h}$ and corresponds to the nodal vector

$$
\mathbf{v}=\left(\begin{array}{c}
\mathbf{v}^{\Gamma} \\
\mathbf{v}^{\Omega}+\mathbf{w}^{\Omega}
\end{array}\right), \quad \text { i.e. } v_{h}=\sum_{j=1}^{N} v_{j} \varphi_{j}[\mathbf{x}] .
$$

Using the fact that $\mathbf{A}_{22}(\mathbf{x})$ is invertible, it is easily seen that the solution $\mathbf{v}$ does not depend on the particular choice of $\mathbf{w}^{\Omega}$, which is why we use $\mathbf{w}^{\Omega}=0$.

The matrix-vector formulation reads (omitting the Kronecker product notation)

$$
\begin{aligned}
\frac{\mathrm{d}}{\mathrm{d} t}(\mathbf{M}(\mathbf{x}) \mathbf{u})+\mathbf{A}(\mathbf{x}) \mathbf{u} & =\mathbf{f}(\mathbf{x}) \\
-\mathbf{A}_{22}(\mathbf{x}) \mathbf{v}^{\Omega}(\mathbf{x}) & =\mathbf{A}_{21}(\mathbf{x}) \mathbf{v}^{\Gamma}(\mathbf{x}) \\
\frac{\mathrm{d}}{\mathrm{d} t}\left(\begin{array}{c}
\mathbf{x}^{\Gamma} \\
\mathbf{x}^{\Omega}
\end{array}\right)=\dot{\mathbf{x}} & =\mathbf{v}=\left(\begin{array}{c}
\mathbf{v}^{\Gamma} \\
\mathbf{v}^{\Omega}
\end{array}\right)
\end{aligned}
$$

The initial nodal vectors $\mathbf{u}(0)$ and $\mathbf{x}(0)$ are chosen as in (3.3).

We will see in the following sections that the matrix-vector formulation is the only tool used in the stability analysis, where geometric estimates are only needed for the consistency analysis.

\subsection{Lifted finite element space}

In the error analysis, we compare functions on three different domains: the exact domain $\Omega(t)$, the discrete domain $\Omega_{h}(t)=\Omega_{h}(\mathbf{x}(t))$ obtained by the finite element method and the interpolated exact domain $\Omega_{h}^{*}(t)=\Omega_{h}\left(\mathbf{x}^{*}(t)\right)$, which is the computational domain corresponding to the nodal vector $\mathbf{x}^{*}(t)$ with the exact positions $x_{j}^{*}(t)=X\left(x_{j}^{0}, t\right)$ of the nodes at time $t$ and only available in theory.

Any finite element function $u_{h} \in S_{h}(\mathbf{x})$ on the discrete computational domain, with nodal values $u_{j}$, $j=1, \ldots, N$, is related to a finite element function $\widehat{u}_{h} \in S_{h}\left(\mathbf{x}^{*}\right)$ with the same nodal values:

$$
\widehat{u}_{h}=\sum_{j=1}^{N} u_{j} \varphi_{j}\left[\mathbf{x}^{*}\right]
$$

Based on Section 3.1, we obtain a map $\Lambda_{h}(\cdot, t): \Omega_{h}\left(\mathbf{x}^{*}(t)\right) \rightarrow \Omega(t)$ (cf. Elliott \& Ranner (2013, 2017)), that is defined element-wise and maps the curved elements of the triangulation of $\Omega_{h}\left(\mathbf{x}^{*}(t)\right)$ onto the corresponding parts of $\Omega(t)$. Restricted to interior simplices with at most one node on the boundary, this map is the identity. On boundary simplices, $\Lambda_{h}$ is of class $C^{k}$ if the boundary is of class $C^{k}$ (see (Elliott \& Ranner, 2013, Lemma 4.6)). 
Definition 3.1 For a function $\widehat{u}_{h} \in S_{h}\left(\mathbf{x}^{*}(t)\right)$, we define its lift $\widehat{u}_{h}^{\ell}: \Omega(t) \rightarrow \mathbb{R}$ by

$$
\widehat{u}_{h}^{\ell}\left(\Lambda_{h}(x, t), t\right):=\widehat{u}_{h}(x, t) .
$$

The composed lift from finite element functions $u_{h}$ on $\Omega_{h}(\mathbf{x}(t))$ to functions on $\Omega(t)$ is denoted by

$$
u_{h}^{L}=\widehat{u}_{h}^{\ell} .
$$

For any $u \in H^{k+1}(\Omega)$, there exists a unique finite element interpolation in the nodes $x_{j}^{*}$, denoted by $\widetilde{I}_{h} u \in S_{h}\left(\mathbf{x}^{*}\right)$. We set $I_{h} u=\left(\widetilde{I}_{h} u\right)^{\ell}: \Omega \rightarrow \mathbb{R}$. An interpolation estimate is obtained from (Elliott \& Ranner, 2013, Proposition 5.4), based on Bernardi (1989).

Proposition 3.2 (Interpolation error) There exists a constant $c>0$ independent of $h \leqslant h_{0}$ ( $h_{0}$ sufficiently small) and $t$ such that for all $1 \leqslant m \leqslant k, u(\cdot, t) \in H^{m+1}(\Omega(t))$ and $t \in[0, T]$

$$
\left\|u-I_{h} u\right\|_{L^{2}(\Omega(t))}+h\left\|\nabla\left(u-I_{h} u\right)\right\|_{L^{2}(\Omega(t))} \leqslant c h^{m}\|u\|_{H^{m+1}(\Omega(t))} .
$$

\section{Statement of the main result}

We are now able to formulate the main result of this paper, which yields error bounds for the spatial semi-discretization of (2.8) with evolving isoparametric finite elements of polynomial degree $k \geqslant 2$. We introduce the notation

$$
x_{h}^{L}(x, t)=X_{h}^{L}(p, t) \in \Omega_{h}(t) \quad \text { for } \quad x=X(p, t) \in \Omega(t) .
$$

THEOREM 4.1 Consider the spatial semi-discretization (3.2) of (2.8) with evolving isoparametric finite elements of order $k \geqslant 2$. We assume a quasi-uniform admissible triangulation of the initial domain and initial values chosen by finite element interpolations of the exact initial data. Assume that the problem admits an exact solution $(u, v, X)$ that is sufficiently smooth $\left(u \in H^{k+1}(\Omega(t)), v, X \in H^{k+1}(\Omega(t))^{n}, n=\right.$ $2,3)$ for $t \in[0, T]$ and a quasi-uniform triangulation of $\Omega_{0}$. Then there exists an $h_{0}>0$ such that for all mesh widths $h \leqslant h_{0}$ the following error bounds hold on $\Omega(t)$, for $t \in[0, T]$ :

$$
\begin{array}{r}
\left(\left\|u_{h}^{L}(\cdot, t)-u(\cdot, t)\right\|_{L^{2}(\Omega(t))}^{2}+\int_{0}^{t}\left\|u_{h}^{L}(\cdot, s)-u(\cdot, s)\right\|_{H^{1}(\Omega(s))^{2}}^{2} \mathrm{~d} s\right)^{\frac{1}{2}} \leqslant c h^{k}, \\
\left\|v_{h}^{L}(\cdot, t)-v(\cdot, t)\right\|_{H^{1}(\Omega(t))^{n}} \leqslant c h^{k}, \\
\left\|X_{h}^{L}(\cdot, t)-X(\cdot, t)\right\|_{H^{1}\left(\Omega_{0}\right)^{n}} \leqslant c h^{k} .
\end{array}
$$

The constant $c$ depends on the regularity of the exact solution $(u, v, X)$, on $T$ and on the regularity of $f$.

In the following proof of error bounds, we clearly separate the stability and consistency analysis. The stability analysis, which is the significantly more difficult task in this work, borrows techniques used in Kovács et al. (2017) and extends them to the present evolving bulk problem. The crucial differences are that in the stability analysis for the domain evolution the boundary has to be taken into account and the error only lives in the interior of the domain, whereas for the diffusion equation there is also an error on the boundary. The stability analysis relies on auxiliary results from Section 5, which require a bound on 
the $W^{1, \infty}$-norm of the position errors. With the $H^{1}$-norm error bound together with an inverse estimate, we obtain an $\mathscr{O}\left(h^{k-n / 2}\right)$ error bound for the position error, which is only small for $k \geqslant 2$. This is why we impose the condition $k \geqslant 2$ in the above result. To apply this inverse estimate, we need that the interpolated exact domain $\Omega_{h}^{*}(t)$, which is the triangulation of $\Omega(t)$ with the nodes $X\left(p_{j}, t\right)$, is quasiuniform. Since the exact flow map $X(\cdot, t): \Omega_{0} \rightarrow \Omega(t)$ is assumed to be smooth and non-degenerate, it is locally close to an invertible linear transformation, and therefore preserves admissibility of meshes on compact time intervals for sufficiently small $h \leqslant h_{0}$, although the bounds in the admissibility inequalities and the largest possible mesh width may deteriorate with growing time. The boundedness of the $W^{1, \infty}$ norm of the position error is ensured with the $\mathscr{O}\left(h^{k}\right)$ error bound in $H^{1}$ norm that yields a $\mathscr{O}\left(h^{k-n / 2}\right)$ bound in the $W^{1, \infty}$ norm with an inverse inequality. Therefore the assumptions of the theorem exclude a degeneration of the mesh for sufficiently small $h_{0}$.

The consistency analysis requires geometric estimates for the evolving isoparametric finite element method. Such estimates are mainly taken from Elliott \& Ranner (2013), which are generalized to the time-dependent case in Elliott \& Ranner (2017).

The stability proof will yield $h$-independent bounds of the errors in terms of the defects. The stability analysis is done in the matrix-vector formulation, which allows a compact and manageable representation of the computations. We use energy estimates and transport formulae to relate mass and stiffness matrices for different nodal vectors. This allows us to work with the interpolated exact domain $\Omega_{h}\left(\mathbf{x}^{*}(t)\right)$, which is a finite element triangulation of $\Omega(t)$ and only available in theoretical consideration.

In Section 5 we prove auxiliary results that are used in the stability analysis, and then collect geometric estimates which are needed for the consistency analysis. In Section 6 we analyze stability of the semi-discrete velocity law without a diffusion equation on the evolving domain. The stability analysis of the semi-discrete diffusion equation, which requires results from Section 6, is then done in Section 7. The defects are then bounded in Section 8 and the proof of Theorem 4.1 is completed in Section 9.

\section{Auxiliary results}

\subsection{Properties of the evolving mass and stiffness matrix}

The following construction and results are similar to (Kovács et al., 2017, Section 4), where similar identities are shown for surfaces only. We extend these results to the present case of domains. In the stability analysis, we have to relate finite element matrices corresponding to different nodal vectors. Let $\mathbf{x}, \mathbf{y} \in \mathbb{R}^{3 N}$ be two nodal vectors defining discrete domains $\Omega_{h}(\mathbf{x}), \Omega_{h}(\mathbf{y})$, respectively. We set $\mathbf{e}=\mathbf{x}-\mathbf{y}$. For any $\theta \in[0,1]$, we have the intermediate domain $\Omega_{h}^{\theta}=\Omega_{h}(\mathbf{y}+\theta \mathbf{e})$ which is the discrete domain corresponding to the intermediate nodal vector $\mathbf{y}+\theta \mathbf{e}$.

For any vector $\mathbf{w} \in \mathbb{R}^{N}$, we set

$$
w_{h}^{\theta}=\sum_{j=1}^{N} w_{j} \varphi_{j}[\mathbf{y}+\theta \mathbf{e}] \in S_{h}(\mathbf{y}+\theta \mathbf{e})
$$

In particular, we have the finite element function $e_{h}^{\theta}$ corresponding to $\mathbf{e}$ :

$$
e_{h}^{\theta}=\sum_{j=1}^{N} e_{j} \varphi_{j}[\mathbf{y}+\theta \mathbf{e}]
$$


LEMMA 5.1 In the above setting, the following identities hold for any $\mathbf{w}, \mathbf{z} \in \mathbb{R}^{N}$ :

$$
\begin{gathered}
\mathbf{w}^{\mathrm{T}}(\mathbf{M}(\mathbf{x})-\mathbf{M}(\mathbf{y})) \mathbf{z}=\int_{0}^{1} \int_{\Omega_{h}^{\theta}} w_{h}^{\theta}\left(\nabla \cdot e_{h}^{\theta}\right) z_{h}^{\theta} \mathrm{d} x \mathrm{~d} \theta, \\
\mathbf{w}^{\mathrm{T}}(\mathbf{A}(\mathbf{x})-\mathbf{A}(\mathbf{y})) \mathbf{z}=\int_{0}^{1} \int_{\Omega_{h}^{\theta}} \nabla w_{h}^{\theta} \cdot\left(D_{\Omega_{h}^{\theta}} e_{h}^{\theta}\right) \nabla z_{h}^{\theta} \mathrm{d} x \mathrm{~d} \theta,
\end{gathered}
$$

where $D_{\Omega_{h}^{\theta}}=\operatorname{trace}\left(\nabla e_{h}^{\theta}\right) I_{3}-\left(\nabla e_{h}^{\theta}+\left(\nabla e_{h}^{\theta}\right)^{\mathrm{T}}\right)$.

Proof. We use transport formulae from (Elliott \& Ranner, 2017, p. 23):

$$
\begin{aligned}
& \mathbf{w}^{\mathrm{T}}(\mathbf{A}(\mathbf{x})-\mathbf{A}(\mathbf{y})) \mathbf{z}=\int_{\Omega_{h}(\mathbf{x})} \nabla w_{h}^{1} \cdot \nabla z_{h}^{1} \mathrm{~d} x-\int_{\Omega_{h}(\mathbf{y})} \nabla w_{h}^{0} \cdot \nabla z_{h}^{0} \mathrm{~d} x=\int_{0}^{1} \frac{\mathrm{d}}{\mathrm{d} \theta} \int_{\Omega_{h}^{\theta}} \nabla w_{h}^{\theta} \cdot \nabla z_{h}^{\theta} \mathrm{d} x \mathrm{~d} \theta \\
& =\int_{0}^{1} \int_{\Omega_{h}^{\theta}} \nabla \partial_{\theta}^{\bullet} w_{h}^{\theta} \cdot \nabla z_{h}^{\theta}+\nabla w_{h}^{\theta} \cdot \nabla \partial_{\theta}^{\bullet} z_{h}^{\theta}+\left(\left(\nabla \cdot e_{h}^{\theta}\right) I_{3}-\left(\nabla e_{h}^{\theta}+\left(\nabla e_{h}^{\theta}\right)^{\mathrm{T}}\right)\right) \nabla w_{h}^{\theta} \cdot \nabla z_{h}^{\theta} \mathrm{d} x \mathrm{~d} \theta .
\end{aligned}
$$

The first two terms vanish thanks to the transport property. This shows the second identity, since $\nabla \cdot e_{h}^{\theta}=$ $\operatorname{trace}\left(\nabla e_{h}^{\theta}\right)$. The first identity is proven similarly.

A direct consequence is the following lemma, where for any symmetric and positive (semi-)definite matrix $\mathbf{K}$, we denote the induced (semi-)norm on $\mathbb{R}^{N}$ by $\|\mathbf{w}\|_{\mathbf{K}}:=\left(w^{\mathrm{T}} \mathbf{K w}\right)^{1 / 2}$.

LEMMA 5.2 If $\left\|\nabla \cdot e_{h}^{\theta}\right\|_{L^{\infty}\left(\Omega_{h}^{\theta}\right)} \leqslant \mu$ for $\theta \in[0,1]$, then

$$
\|\mathbf{w}\|_{\mathbf{M}(\mathbf{y}+\theta \mathbf{e})} \leqslant e^{\frac{\mu}{2}}\|\mathbf{w}\|_{\mathbf{M}(\mathbf{y})} \text { for } \theta \in[0,1] .
$$

If $\left\|D_{\Omega_{h}^{\theta}} e_{h}^{\theta}\right\|_{L^{\infty}\left(\Omega_{h}^{\theta}\right)} \leqslant \eta$ for $\theta \in[0,1]$, then

$$
\|\mathbf{w}\|_{\mathbf{A}(\mathbf{y}+\theta \mathbf{e})} \leqslant e^{\frac{\eta}{2}}\|\mathbf{w}\|_{\mathbf{A}(\mathbf{y})} \text { for } \theta \in[0,1] .
$$

Proof. We use the previous lemma and an $L^{2}-L^{\infty}-L^{2}$-estimate and compute for $0 \leqslant \tau \leqslant 1$ :

$$
\begin{aligned}
\|\mathbf{w}\|_{\mathbf{M}(\mathbf{y}+\tau \mathbf{e})}^{2}-\|\mathbf{w}\|_{\mathbf{M}(\mathbf{y})}^{2} & =\mathbf{w}^{\mathrm{T}}(\mathbf{M}(\mathbf{y}+\tau \mathbf{e})-\mathbf{M}(\mathbf{y})) \mathbf{w} \\
& =\int_{0}^{\tau} \int_{\Omega_{h}^{\theta}} w_{h}^{\theta} \nabla \cdot e_{h}^{\theta} w_{h}^{\theta} \mathrm{d} x \mathrm{~d} \theta \\
& =\int_{0}^{\tau}\|\mathbf{w}\|_{\mathbf{M}(\mathbf{y}+\theta \mathbf{e})}^{2}\left\|\nabla \cdot e_{h}^{\theta}\right\|_{L^{\infty}\left(\Omega_{h}^{\theta}\right)} \mathrm{d} \theta \\
& \leqslant \mu \int_{0}^{\tau}\|\mathbf{w}\|_{\mathbf{M}(\mathbf{y}+\theta \mathbf{e})}^{2} \mathrm{~d} \theta .
\end{aligned}
$$

A Gronwall argument shows the first result. The second estimate is shown analogously.

LEMMA 5.3 Assume that

$$
\left\|\nabla e_{h}^{0}\right\|_{L^{\infty}\left(\Omega_{h}(\mathbf{y})\right)} \leqslant \frac{1}{2} .
$$

Then, for $0 \leqslant \theta \leqslant 1$ the function $w_{h}^{\theta}=\sum_{j=1}^{N} w_{j} \varphi_{j}[\mathbf{y}+\theta \mathbf{e}]$ on $\Omega_{h}^{\theta}$ is bounded by

$$
\left\|\nabla w_{h}^{\theta}\right\|_{L^{p}\left(\Omega_{h}^{\theta}\right)} \leqslant c_{p}\left\|\nabla w_{h}^{0}\right\|_{L^{p}\left(\Omega_{h}^{0}\right)},
$$




$$
\left\|w_{h}^{\theta}\right\|_{L^{p}\left(\Omega_{h}^{\theta}\right)} \leqslant \widetilde{c}_{p}\left\|w_{h}^{0}\right\|_{L^{p}\left(\Omega_{h}^{0}\right)},
$$

for $1 \leqslant p \leqslant \infty$, where $c_{p}$ and $\widetilde{c}_{p}$ depend only on $p$.

Proof. We parametrize $\Omega_{h}^{\theta}$ over $\Omega_{h}^{0}$ :

$$
\begin{aligned}
Y_{h}^{\theta}\left(p_{h}\right) & =Y_{h}\left(p_{h}, \theta\right)=\sum_{j=1}^{N}\left(y_{j}+\theta e_{j}\right) \varphi_{j}[\mathbf{y}]\left(p_{h}\right) \quad\left(p_{h} \in \Omega_{h}^{0}=\Omega_{h}(\mathbf{y})\right) \\
& =\sum_{j=1}^{N} y_{j} \varphi_{j}[\mathbf{y}]\left(p_{h}\right)+\theta \sum_{j=1}^{N} e_{j} \varphi_{j}[\mathbf{y}]\left(p_{h}\right)=p_{h}+\theta e_{h}^{0}\left(p_{h}\right)
\end{aligned}
$$

where we have used that $Y_{h}^{0}\left(p_{h}\right)=p_{h}$. Differentiating with respect to $p_{h}$ yields

$$
D Y_{h}^{\theta}\left(p_{h}\right)=I+\theta D e_{h}^{0}\left(p_{h}\right) .
$$

By the transport property, we have $w_{h}^{\theta}\left(Y_{h}^{\theta}\left(p_{h}\right)\right)=w_{h}^{0}\left(Y_{h}^{0}\left(p_{h}\right)\right)=w_{h}^{0}\left(p_{h}\right)$. Differentiation with respect to $p_{h}$ yields

$$
D w_{h}^{\theta}\left(Y_{h}^{\theta}\left(p_{h}\right)\right) D Y_{h}^{\theta}\left(p_{h}\right)=D w_{h}^{0}\left(p_{h}\right)
$$

From (5.2) we have under the assumption $\left\|\nabla e_{h}^{0}\right\|_{L^{\infty}\left(\Omega_{h}(\mathbf{y})\right)} \leqslant \frac{1}{2}$ :

$$
\left|D Y_{h}^{\theta}\left(p_{h}\right) z\right|=\left|z+\theta\left(\nabla e_{h}^{0}\right)^{\mathrm{T}} z\right| \geqslant|z|-\theta\left|\left(\nabla e_{h}^{0}\right)^{\mathrm{T}} z\right| \geqslant \frac{1}{2}|z| .
$$

Thus, the matrix $D Y_{h}^{\theta}\left(p_{h}\right)$ is invertible and we have with (5.3)

$$
D w_{h}^{\theta}\left(Y_{h}^{\theta}\left(p_{h}\right)\right)=D w_{h}^{0}\left(p_{h}\right)\left(D Y_{h}^{\theta}\left(p_{h}\right)\right)^{-1}
$$

implying $\left|D w_{h}^{\theta}\left(Y_{h}^{\theta}\left(p_{h}\right)\right)\right| \leqslant 2\left|D w_{h}^{0}\left(p_{h}\right)\right|$ and thus

$$
\left\|\nabla w_{h}^{\theta}\right\|_{L^{\infty}\left(\Omega_{h}^{\theta}\right)} \leqslant 2\left\|\nabla w_{h}^{0}\right\|_{L^{\infty}\left(\Omega_{h}^{0}\right)} .
$$

For $1 \leqslant p<\infty$, we use the transformation formula and the fact that $\left\|D e_{h}^{0}\left(p_{h}\right)\right\|_{L^{\infty}\left(\Omega_{h}^{0}\right)} \leqslant \frac{1}{2}$ to obtain

$$
\begin{aligned}
\left\|\nabla w_{h}^{\theta}\right\|_{L^{p}\left(\Omega_{h}^{\theta}\right)}^{p} & =\int_{\Omega_{h}^{\theta}}\left|D w_{h}^{\theta}\left(y_{h}^{\theta}\right)\right|^{p} \mathrm{~d} y_{h}^{\theta}=\int_{\Omega_{h}^{0}}\left|D w_{h}^{\theta}\left(Y_{h}^{\theta}\left(p_{h}\right)\right)\right|^{p}\left|\operatorname{det} D Y_{h}^{\theta}\left(p_{h}\right)\right| \mathrm{d} p_{h} \\
& =\int_{\Omega_{h}^{0}}\left|D w_{h}^{0}\left(p_{h}\right)\left(D Y_{h}^{\theta}\left(p_{h}\right)\right)^{-1}\right|^{p}\left|\operatorname{det} D Y_{h}\left(p_{h}\right)\right| \mathrm{d} p_{h} \\
& \leqslant c \int_{\Omega_{h}^{0}}\left|D w_{h}^{0}\left(p_{h}\right)\right|^{p} \mathrm{~d} p_{h}=c\left\|\nabla w_{h}^{0}\right\|_{L^{p}\left(\Omega_{h}^{0}\right)}^{p} .
\end{aligned}
$$

For the second estimate, we note that the transport property immediately implies

$$
\left\|w_{h}^{\theta}\right\|_{L^{\infty}\left(\Omega_{h}^{\theta}\right)}=\left\|w_{h}^{0}\right\|_{L^{\infty}\left(\Omega_{h}^{0}\right)} .
$$

For $1 \leqslant p<\infty$, we use the transformation formula and the same arguments as above.

Another consequence of Lemma 5.2 is the following. 
Lemma 5.4 Let $\mathbf{x}^{*}(t)$ be the vector of the exact positions $x_{j}^{*}(t)=X\left(x_{j}^{0}, t\right)$. Then, we have for all $\mathbf{w}, \mathbf{z} \in \mathbb{R}^{N}:$

$$
\begin{gathered}
\mathbf{w}^{\mathrm{T}}\left(\frac{\mathrm{d}}{\mathrm{d} t} \mathbf{M}\left(\mathbf{x}^{*}(t)\right)\right) \mathbf{z} \leqslant c\|\mathbf{w}\|_{\mathbf{M}\left(\mathbf{x}^{*}(t)\right)}\|\mathbf{z}\|_{\mathbf{M}\left(\mathbf{x}^{*}(t)\right)}, \\
\mathbf{w}^{\mathrm{T}}\left(\frac{\mathrm{d}}{\mathrm{d} t} \mathbf{A}\left(\mathbf{x}^{*}(t)\right)\right) \mathbf{z} \leqslant c\|\mathbf{w}\|_{\mathbf{A}\left(\mathbf{x}^{*}(t)\right)}\|\mathbf{z}\|_{\mathbf{A}\left(\mathbf{x}^{*}(t)\right)} .
\end{gathered}
$$

The constant $c$ depends on the $W^{1, \infty}\left(\Omega_{T}\right)$-norm of $v$ the dimension $n$ and the length $T$ of the time interval, but is independent of $h$ and $t$.

Proof. The proof can be found in (Dziuk et al., 2012, Lemma 4.1) for surfaces and can directly be transferred to the present situation, using arguments from the proof of Lemma 5.2.

\subsection{Geometric estimates}

We collect geometric estimates that are used later in the consistency analysis. For a finite element function $\eta_{h}: \Omega_{h}^{*}(t) \rightarrow \mathbb{R}$, its lift is denoted by $\eta_{h}^{\ell}: \Omega(t) \rightarrow \mathbb{R}$ (see Definition 3.1). The following lemma shows that the norms of finite element functions and their lifts are equivalent. A proof can be found in (Elliott \& Ranner, 2013, Proposition 4.9), based on Ciarlet \& Raviart (1972).

Lemma 5.5 Let $\eta_{h}: \Omega_{h}^{*}(t) \rightarrow \mathbb{R}$ with lift $\eta_{h}^{\ell}: \Omega(t) \rightarrow \mathbb{R}$. Then there exist constants $c_{1}, c_{2}>0$ such that

$$
\begin{aligned}
c_{1}\left\|\eta_{h}\right\|_{L^{2}\left(\Omega_{h}^{*}(t)\right)} & \leqslant\left\|\eta_{h}^{\ell}\right\|_{L^{2}(\Omega(t))} \leqslant c_{2}\left\|\eta_{h}\right\|_{L^{2}\left(\Omega_{h}^{*}(t)\right)}, \\
c_{1}\left\|\nabla \eta_{h}\right\|_{L^{2}\left(\Omega_{h}^{*}(t)\right)} & \leqslant\left\|\nabla \eta_{h}^{\ell}\right\|_{L^{2}(\Omega(t))} \leqslant c_{2}\left\|\nabla \eta_{h}\right\|_{L^{2}\left(\Omega_{h}^{*}(t)\right)} .
\end{aligned}
$$

The constant $c$ depends on the dimension $n$, the length $T$ of the time interval and the geometry of $\Omega_{T}$ but is independent of $h$ and $t$.

We define discrete analogues of the bilinear forms $m$ and $a$, defined in (2.3): For $\eta_{h}, \chi_{h}: \Omega_{h}^{*}(t) \rightarrow \mathbb{R}$, we define

$$
\begin{aligned}
& m_{h}^{*}\left(\eta_{h}, \chi_{h}\right)=\int_{\Omega_{h}^{*}(t)} \eta_{h} \chi_{h}, \\
& a_{h}^{*}\left(\eta_{h}, \chi_{h}\right)=\int_{\Omega_{h}^{*}(t)} \nabla \eta_{h} \cdot \nabla \chi_{h} .
\end{aligned}
$$

The following lemma estimates the difference between the discrete bilinear form on the interpolated exact domain and the exact bilinear form of the lifted functions on the exact domain. A proof can be found in Elliott \& Ranner (2017).

Lemma 5.6 (Geometric approximation errors) For $\eta_{h}, \chi_{h} \in S_{h}\left(\mathbf{x}^{*}(t)\right)$ with corresponding lifts $\eta_{h}^{\ell}, \chi_{h}^{\ell}$, the following estimates hold: there exists a constant $c$ such that

$$
\begin{aligned}
&\left|m_{h}^{*}\left(\eta_{h}, \chi_{h}\right)-m\left(\eta_{h}^{\ell}, \chi_{h}^{\ell}\right)\right| \leqslant c h^{k}\left\|\eta_{h}^{\ell}\right\|_{L^{2}(\Omega(t))}\left\|\chi_{h}^{\ell}\right\|_{L^{2}(\Omega(t))}, \\
&\left|a_{h}^{*}\left(\eta_{h}, \chi_{h}\right)-a\left(\eta_{h}^{\ell}, \chi_{h}^{\ell}\right)\right| \leqslant c h^{k}\left\|\nabla \eta_{h}^{\ell}\right\|_{L^{2}(\Omega(t))}\left\|\nabla \chi_{h}^{\ell}\right\|_{L^{2}(\Omega(t))} .
\end{aligned}
$$

The constant $c$ depends on the dimension $n$, the length $T$ of the interval and the geometry of $\Omega_{T}$ but is independent of $h$ and $t$. 


\section{Stability of the semi-discrete harmonic velocity law}

We will start with analyzing stability of the semi-discrete velocity law without the diffusion equation, since the domain evolution is independent of the parabolic equation, see Remark 2.1. The stability analysis of the semi-discrete diffusion equation, which is based on the following results, is presented in the next section.

We consider the nodal vectors $\mathbf{v}, \mathbf{x} \in \mathbb{R}^{3 N}$ which satisfy

$$
\begin{gathered}
\left(I_{3} \otimes \mathbf{A}_{22}(\mathbf{x})\right) \mathbf{v}^{\Omega}=-\left(I_{3} \otimes \mathbf{A}_{21}(\mathbf{x})\right) \mathbf{v}^{\Gamma}, \\
\dot{\mathbf{x}}=\mathbf{v} .
\end{gathered}
$$

with given $\mathbf{v}^{\Gamma}$. We denote by

$$
\mathbf{x}^{*}(t)=\left(\begin{array}{l}
\mathbf{x}^{\Gamma, *}(t) \\
\mathbf{x}^{\Omega, *}(t)
\end{array}\right)
$$

the vector of the exact positions at time $t \in[0, T]$. Note that $x_{j}^{*}(t)=x_{j}(t)$ for all $j=1, \ldots, N_{\Gamma}$ since $\mathbf{v}^{\Gamma}$ is given explicitly. i.e. $\mathbf{x}^{\Gamma}(t)=\mathbf{x}^{\Gamma, *}(t)$.

We consider the interpolated exact velocity $v_{h}^{*}(\cdot, t)=\sum_{j=1}^{N} v_{j}^{*}(t) \varphi_{j}\left[\mathbf{x}^{*}(t)\right]$ with the corresponding nodal vector

$$
\mathbf{v}^{*}(t)=\left(\begin{array}{c}
\mathbf{v}^{\Gamma, *}(t) \\
\mathbf{v}^{\Omega, *}(t)
\end{array}\right) .
$$

Note again that $\mathbf{v}^{\Gamma, *}(t)=\mathbf{v}^{\Gamma}(t)$.

\subsection{Error equations}

The vectors $\mathbf{x}^{*}$ and $\mathbf{v}^{*}$ satisfy (6.1) up to a defect $\mathbf{d}_{\mathbf{v} \Omega}$ :

$$
\begin{aligned}
\left(I_{3} \otimes \mathbf{A}_{22}\left(\mathbf{x}^{*}\right)\right) \mathbf{v}^{\Omega, *} & =-\left(I_{3} \otimes \mathbf{A}_{21}\left(\mathbf{x}^{*}\right)\right) \mathbf{v}^{\Gamma, *}+\mathbf{M}_{22}\left(\mathbf{x}^{*}\right) \mathbf{d}_{\mathbf{v}} \Omega, \\
\dot{\mathbf{x}}^{*} & =\mathbf{v}^{*} .
\end{aligned}
$$

We set $\mathbf{d}_{\mathbf{v}}=\left(\mathbf{d}_{\mathbf{v}} \Gamma, \mathbf{d}_{\mathbf{v}} \Omega\right) \in \mathbb{R}^{3 N}$ with $\mathbf{d}_{\mathbf{v} \Gamma}=0 \in \mathbb{R}^{3 N_{\Gamma}}$. This notation will be useful in the stability analysis. The defect $\mathbf{d}_{\mathbf{v}}$ corresponds to a finite element function $d_{h}^{v}(\cdot, t)=\sum_{j=1}^{N} d_{j}^{v}(t) \varphi_{j}\left[\mathbf{x}^{*}(t)\right] \in S_{0, h}(\mathbf{x}(t))^{3}$. We denote the errors in the nodes and in the velocity by $\mathbf{e}_{\mathbf{x}^{\Omega}}=\mathbf{x}^{\Omega}-\mathbf{x}^{\Omega, *}, \mathbf{e}_{\mathbf{v}^{\Omega}}=\mathbf{v}^{\Omega}-\mathbf{v}^{\Omega, *}$ and use the notation

$$
\mathbf{e}_{\mathbf{x}}=\left(\begin{array}{c}
0 \\
\mathbf{e}_{\mathbf{x}^{\Omega}}
\end{array}\right)=\left(\begin{array}{c}
\mathbf{e}_{\mathbf{x}} \Gamma \\
\mathbf{e}_{\mathbf{x}^{\Omega}}
\end{array}\right), \quad \mathbf{e}_{\mathbf{v}}=\left(\begin{array}{c}
0 \\
\mathbf{e}_{\mathbf{v} \Omega}
\end{array}\right)=\left(\begin{array}{c}
\mathbf{e}_{\mathbf{v}} \Gamma \\
\mathbf{e}_{\mathbf{v} \Omega}
\end{array}\right) .
$$

In the following, we write $\mathbf{A}(\mathbf{x})$ instead of $I_{3} \otimes \mathbf{A}(\mathbf{x})$, for brevity. We rewrite (6.1) as

$$
\mathbf{A}_{22}\left(\mathbf{x}^{*}\right) \mathbf{v}^{\Omega}=-\left(\mathbf{A}_{22}(\mathbf{x})-\mathbf{A}_{22}\left(\mathbf{x}^{*}\right)\right) \mathbf{v}^{\Omega, *}-\left(\mathbf{A}_{22}(\mathbf{x})-\mathbf{A}_{22}\left(\mathbf{x}^{*}\right)\right) \mathbf{e}_{\mathbf{v}} \Omega-\mathbf{A}_{21}(\mathbf{x}) \mathbf{v}^{\Gamma} .
$$

Subtracting (6.2) from (6.3) and using $\mathbf{v}^{\Gamma}=\mathbf{v}^{\Gamma, *}$ yields the error equations

$$
\begin{aligned}
\mathbf{A}_{22}\left(\mathbf{x}^{*}\right) \mathbf{e}_{\mathbf{v}} \Omega= & -\left(\mathbf{A}_{22}(\mathbf{x})-\mathbf{A}_{22}\left(\mathbf{x}^{*}\right)\right) \mathbf{v}^{\Omega, *}-\left(\mathbf{A}_{22}(\mathbf{x})-\mathbf{A}_{22}\left(\mathbf{x}^{*}\right)\right) \mathbf{e}_{\mathbf{v}} \Omega \\
& -\left(\mathbf{A}_{21}(\mathbf{x})-\mathbf{A}_{21}\left(\mathbf{x}^{*}\right)\right) \mathbf{v}^{\Gamma, *}-\mathbf{M}_{22}\left(\mathbf{x}^{*}\right) \mathbf{d}_{\mathbf{v}} \Omega \\
\dot{\mathbf{e}}_{\mathbf{x}^{\Omega}=} & \mathbf{e}_{\mathbf{v} \Omega}
\end{aligned}
$$




\subsection{Dual norms}

We recall that the mass and stiffness matrices $\mathbf{M}_{22}(\mathbf{x})$ and $\mathbf{A}_{22}(\mathbf{x})$, respectively, induce norms on $S_{0, h}(\mathbf{x})$. Note that $\mathbf{A}_{22}(\mathbf{x})$ defines a norm on $S_{0, h}(\mathbf{x})$, whereas $\mathbf{A}(\mathbf{x})$ defines only a semi-norm on $S_{h}(\mathbf{x})$. We define the dual norm

$$
\begin{aligned}
\left\|d_{h}^{v}\right\|_{H_{0, h}^{-1}\left(\Omega_{h}\left(\mathbf{x}^{*}\right)\right)} & =\sup _{0 \neq \psi_{h} \in S_{0, h}\left(\mathbf{x}^{*}\right)^{3}} \frac{\int_{\Omega_{h}\left(\mathbf{x}^{*}\right)} d_{h}^{v} \cdot \psi_{h} \mathrm{~d} x}{\left\|\psi_{h}\right\|_{H_{0}^{1}\left(\Omega_{h}\left(\mathbf{x}^{*}\right)\right)}}=\sup _{0 \neq \mathbf{z} \in \mathbb{R}^{3 N_{\Omega}}} \frac{\mathbf{d}_{\mathbf{v} \Omega}^{\mathrm{T}} \mathbf{M}_{22}\left(\mathbf{x}^{*}\right) \mathbf{z}}{\left(\mathbf{z}^{\mathrm{T}} \mathbf{A}_{22}\left(\mathbf{x}^{*}\right) \mathbf{z}\right)^{1 / 2}} \\
& =\sup _{0 \neq \mathbf{w} \in \mathbb{R}^{3 N_{\Omega}}} \frac{\mathbf{d}_{\mathbf{v}^{\Omega} \Omega}^{\mathrm{T}} \mathbf{M}_{22}\left(\mathbf{x}^{*}\right) \mathbf{A}_{22}\left(\mathbf{x}^{*}\right)^{-1 / 2} \mathbf{w}}{\left(\mathbf{w}^{\mathrm{T}} \mathbf{w}\right)^{1 / 2}}=\left\|\mathbf{A}_{22}\left(\mathbf{x}^{*}\right)^{-1 / 2} \mathbf{M}_{22}\left(\mathbf{x}^{*}\right) \mathbf{d}_{\mathbf{v} \Omega}\right\|_{2} \\
& =\left(\mathbf{d}_{\mathbf{v} \Omega}^{\mathrm{T}} \mathbf{M}_{22}\left(\mathbf{x}^{*}\right) \mathbf{A}_{22}\left(\mathbf{x}^{*}\right)^{-1} \mathbf{M}_{22}\left(\mathbf{x}^{*}\right) \mathbf{d}_{\mathbf{v} \Omega}\right)^{\frac{1}{2}}=:\left\|\mathbf{d}_{\mathbf{v} \Omega}\right\|_{\star, \mathbf{x}^{*}} .
\end{aligned}
$$

\subsection{Stability estimate}

We are now ready to state and prove the first main stability result. The following stability result holds under a smallness assumption on the defect. It will be proven in Section 8 that this assumption is satisfied for $\kappa=k \geqslant 2$, where $k$ is the order of the finite element method.

LEMMA 6.1 Assume that, for some $\kappa>\frac{3}{2}$, the defect is bounded as follows:

$$
\left\|\mathbf{d}_{\mathbf{v}^{\Omega}}(t)\right\|_{\star, \mathbf{x}^{*}(t)} \leqslant c h^{\kappa}, \quad t \in[0, T] .
$$

Then there exists an $h_{0}>0$ such that for $h \leqslant h_{0}$ and $t \in[0, T]$, the following error bounds hold.

$$
\begin{aligned}
& \left\|\mathbf{e}_{\mathbf{x}^{\Omega}}(t)\right\|_{\mathbf{A}_{22}\left(\mathbf{x}^{*}(t)\right)}^{2} \leqslant c \int_{0}^{t} \| \mathbf{d}_{\mathbf{v}^{\Omega}(s) \|_{\star, \mathbf{x}^{*}(s)}^{2} \mathrm{~d} s,} \\
& \left\|\mathbf{e}_{\mathbf{v} \Omega}(t)\right\|_{\mathbf{A}_{22}\left(\mathbf{x}^{*}(t)\right)}^{2} \leqslant c\left\|\mathbf{d}_{\mathbf{v} \Omega}(t)\right\|_{\star, \mathbf{x}^{*}(t)}^{2}+c \int_{0}^{t}\left\|\mathbf{d}_{\mathbf{v} \Omega}(s)\right\|_{\star, \mathbf{x}^{*}(s)}^{2} \mathrm{~d} s .
\end{aligned}
$$

Proof. The proof uses energy estimates that are similar to techniques used in Kovács et al. (2017) and Kovács et al. (2019b). We extend their results for coupled surface problems to the present evolving bulk problem. Since the structure of the proof is similar to the cited works, we might skip some nontrivial steps. However there are some crucial differences that need to be pointed out: The evolving bulk $\Omega(t)$ has a boundary $\Gamma(t)$ that has to be taken into account, whereas in Kovács et al. (2017, 2019b) the considered evolving surfaces have no boundaries or interiors. Moreover, we exploit the fact there is no position or velocity error in the boundary because the boundary velocity is given. This implies that the lift of the finite element function corresponding to the error is a $H_{0}^{1}$-function and turns out to be crucial to estimate the error equations, see (6.11) and (6.12) below. In addition, the space dimension $n \in\{2,3\}$ requires the assumption $\kappa>n / 2$, which is due to an inverse estimate at the end of this proof, see Remark 6.1.

In view of the auxiliary results from Section 5 and in particular condition (5.1), we need to control the $W^{1, \infty}$-norm of the position error $e_{x}(\cdot, t)$. Let $0<t^{*} \leqslant T$ be the maximal time such that

$$
\left\|\nabla e_{x}(\cdot, t)\right\|_{L^{\infty}\left(\Omega_{h}\left(\mathbf{x}^{*}(t)\right)\right)} \leqslant h^{(\kappa-3 / 2) / 2} .
$$

Note that $e_{x}(\cdot, 0)=0$ implies $t^{*}>0$. We prove the stated error bounds for $t \in\left[0, t^{*}\right]$ and then show that $t^{*}=T$. 
We test the first equation of (6.4) with $\mathbf{e}_{\mathbf{v}} \Omega$ and obtain

$$
\begin{aligned}
\left\|\mathbf{e}_{\mathbf{v}} \Omega\right\|_{\mathbf{A}_{22}\left(\mathbf{x}^{*}\right)}^{2}= & -\mathbf{e}_{\mathbf{v} \Omega}^{\mathrm{T}}\left(\mathbf{A}_{22}(\mathbf{x})-\mathbf{A}_{22}\left(\mathbf{x}^{*}\right)\right) \mathbf{v}^{\Omega, *}-\mathbf{e}_{\mathbf{v} \Omega}^{\mathrm{T}}\left(\mathbf{A}_{22}(\mathbf{x})-\mathbf{A}_{22}\left(\mathbf{x}^{*}\right)\right) \mathbf{e}_{\mathbf{v} \Omega} \\
& -\mathbf{e}_{\mathbf{v} \Omega}^{\mathrm{T}}\left(\mathbf{A}_{21}(\mathbf{x})-\mathbf{A}_{21}\left(\mathbf{x}^{*}\right)\right) \mathbf{v}^{\Gamma, *}-\mathbf{e}_{\mathbf{v} \Omega}^{\mathrm{T}} \mathbf{M}_{22}\left(\mathbf{x}^{*}\right) \mathbf{d}_{\mathbf{v}} \Omega
\end{aligned}
$$

It is crucial to combine the first and third term of (6.10). Note that

$$
\begin{aligned}
\left(0, \mathbf{e}_{\mathbf{v} \Omega}^{\mathrm{T}}\right) \mathbf{A}(\mathbf{x})\left(\begin{array}{c}
\mathbf{v}^{\Gamma} \\
\mathbf{v}^{\Omega}
\end{array}\right) & =\left(0, \mathbf{e}_{\mathbf{v}^{\mathrm{T}}}^{\mathrm{T}}\right)\left(\begin{array}{ll}
\mathbf{A}_{11}(\mathbf{x}) & \mathbf{A}_{12}(\mathbf{x}) \\
\mathbf{A}_{21}(\mathbf{x}) & \mathbf{A}_{22}(\mathbf{x})
\end{array}\right)\left(\begin{array}{c}
\mathbf{v}^{\Gamma} \\
\mathbf{v}^{\Omega}
\end{array}\right) \\
& =\mathbf{e}_{\mathbf{v} \Omega}^{\mathrm{T}} \mathbf{A}_{21}(\mathbf{x}) \mathbf{v}^{\Gamma}+\mathbf{e}_{\mathbf{v} \Omega}^{\mathrm{T}} \mathbf{A}_{22}(\mathbf{x}) \mathbf{v}^{\Omega} .
\end{aligned}
$$

We set $\mathbf{e}_{\mathbf{v} \Gamma}=0$ and $\mathbf{e}_{\mathbf{v}}^{\mathrm{T}}=\left(\mathbf{e}_{\mathbf{v} \Gamma}^{\mathrm{T}}, \mathbf{e}_{\mathbf{v} \Omega}^{\mathrm{T}}\right)$. Applying (6.11) to (6.10), we obtain

$$
\begin{aligned}
\left\|\mathbf{e}_{\mathbf{v}}\right\|_{\mathbf{A}\left(\mathbf{x}^{*}\right)}^{2} & =-\mathbf{e}_{\mathbf{v}}^{\mathrm{T}}\left(\mathbf{A}(\mathbf{x})-\mathbf{A}\left(\mathbf{x}^{*}\right)\right) \mathbf{v}^{*}-\mathbf{e}_{\mathbf{v} \Omega}^{\mathrm{T}}\left(\mathbf{A}_{22}(\mathbf{x})-\mathbf{A}_{22}\left(\mathbf{x}^{*}\right)\right) \mathbf{e}_{\mathbf{v} \Omega}-\mathbf{e}_{\mathbf{v} \Omega}^{\mathrm{T}} \mathbf{M}_{22}\left(\mathbf{x}^{*}\right) \mathbf{d}_{\mathbf{v} \Omega} \\
& =-\mathbf{e}_{\mathbf{v}}^{\mathrm{T}}\left(\mathbf{A}(\mathbf{x})-\mathbf{A}\left(\mathbf{x}^{*}\right)\right) \mathbf{v}^{*}-\mathbf{e}_{\mathbf{v}}^{\mathrm{T}}\left(\mathbf{A}(\mathbf{x})-\mathbf{A}\left(\mathbf{x}^{*}\right)\right) \mathbf{e}_{\mathbf{v}}-\mathbf{e}_{\mathbf{v}}^{\mathrm{T}} \mathbf{M}\left(\mathbf{x}^{*}\right) \mathbf{d}_{\mathbf{v}} .
\end{aligned}
$$

We estimate these three terms separately.

(i) We use that

$$
D_{\Omega_{h}^{\theta}} e_{x}^{\theta}=\operatorname{trace}\left(\nabla e_{x}^{\theta}\right) I_{3}-\left(\nabla e_{x}^{\theta}+\left(\nabla e_{x}^{\theta}\right)^{\mathrm{T}}\right)
$$

and thus $\left\|D_{\Omega_{h}^{\theta}} e_{x}^{\theta}\right\| \leqslant c\left\|\nabla e_{x}^{\theta}\right\|$. With Lemma 5.1, an $L^{2}-L^{2}-L^{\infty}$-estimate and Lemma 5.2, we arrive at

$$
\begin{aligned}
\mathbf{e}_{\mathbf{v}}^{\mathrm{T}}\left(\mathbf{A}(\mathbf{x})-\mathbf{A}\left(\mathbf{x}^{*}\right)\right) \mathbf{v}^{*} & =\int_{0}^{1} \int_{\Omega_{h}^{\theta}} \nabla e_{v}^{\theta} \cdot\left(D_{\Omega_{h}^{\theta}} e_{x}^{\theta}\right) \nabla v_{h}^{*, \theta} \mathrm{d} x \mathrm{~d} \theta \\
& \leqslant \int_{0}^{1}\left\|\nabla e_{v}^{\theta}\right\|_{L^{2}\left(\Omega_{h}^{\theta}\right)}\left\|D_{\Omega_{h}^{\theta}} e_{x}^{\theta}\right\|_{L^{2}\left(\Omega_{h}^{\theta}\right)}\left\|\nabla v_{h}^{*, \theta}\right\|_{L^{\infty}\left(\Omega_{h}^{\theta}\right)} \mathrm{d} \theta \\
& \leqslant c\left\|\nabla e_{v}^{0}\right\|_{L^{2}\left(\Omega_{h}^{0}\right)}\left\|\nabla e_{x}^{0}\right\|_{L^{2}\left(\Omega_{h}^{0}\right)}\left\|\nabla v_{h}^{*, 0}\right\|_{L^{\infty}\left(\Omega_{h}^{0}\right)} \\
& =c\left\|\mathbf{e}_{\mathbf{v}}\right\|_{\mathbf{A}\left(\mathbf{x}^{*}\right)}\left\|\mathbf{e}_{\mathbf{x}}\right\|_{\mathbf{A}\left(\mathbf{x}^{*}\right)}\left\|\nabla v_{h}^{*}\right\|_{L^{\infty}\left(\Omega_{h}\left(\mathbf{x}^{*}\right)\right)} .
\end{aligned}
$$

The last factor is bounded by a constant independent of $h$, since $v_{h}^{*}$ is the finite element interpolation of the exact velocity (see (Bernardi, 1989, Theorem 4.1)). Using Young's inequality together with the fact that $\left\|\mathbf{e}_{\mathbf{v}}\right\|_{\mathbf{A}\left(\mathbf{x}^{*}\right)}=\left\|\mathbf{e}_{\mathbf{v} \Omega}\right\|_{\mathbf{A}_{22}\left(\mathbf{x}^{*}\right)}$, we obtain

$$
\mathbf{e}_{\mathbf{v}}^{\mathrm{T}}\left(\mathbf{A}(\mathbf{x})-\mathbf{A}\left(\mathbf{x}^{*}\right)\right) \mathbf{v}^{*} \leqslant \frac{1}{4}\left\|\mathbf{e}_{\mathbf{v} \Omega}\right\|_{\mathbf{A}_{22}\left(\mathbf{x}^{*}\right)}^{2}+C\left\|\mathbf{e}_{\mathbf{x}^{\Omega}}\right\|_{\mathbf{A}_{22}\left(\mathbf{x}^{*}\right)}^{2} .
$$

(ii) Similarly, using the smallness assumption (6.9), we obtain

$$
\begin{aligned}
\mathbf{e}_{\mathbf{v}}^{\mathrm{T}}\left(\mathbf{A}(\mathbf{x})-\mathbf{A}\left(\mathbf{x}^{*}\right)\right) \mathbf{e}_{\mathbf{v}} & \leqslant c\left\|\nabla e_{v}^{0}\right\|_{L^{2}\left(\Omega_{h}\left(\mathbf{x}^{*}\right)\right)}^{2}\left\|\nabla e_{x}^{0}\right\|_{L^{\infty}\left(\Omega_{h}\left(\mathbf{x}^{*}\right)\right)} \\
& \leqslant c h^{(\kappa-3 / 2) / 2}\left\|\mathbf{e}_{\mathbf{v}}\right\|_{\mathbf{A}\left(\mathbf{x}^{*}\right)}^{2}=c h^{(\kappa-3 / 2) / 2}\left\|\mathbf{e}_{\mathbf{v} \Omega}\right\|_{\mathbf{A}_{22}\left(\mathbf{x}^{*}\right)}^{2} .
\end{aligned}
$$

(iii) Using the Cauchy-Schwarz inequality together with Young's inequality, we estimate

$$
\begin{aligned}
\mathbf{e}_{\mathbf{v} \Omega}^{\mathrm{T}} \mathbf{M}_{22}\left(\mathbf{x}^{*}\right) \mathbf{d}_{\mathbf{v} \Omega} & =\mathbf{e}_{\mathbf{v} \Omega}^{\mathrm{T}} \mathbf{A}_{22}\left(\mathbf{x}^{*}\right)^{\frac{1}{2}} \mathbf{A}_{22}\left(\mathbf{x}^{*}\right)^{-\frac{1}{2}} \mathbf{M}_{22}\left(\mathbf{x}^{*}\right) \mathbf{d}_{\mathbf{v} \Omega} \\
& \leqslant \frac{1}{4}\left\|\mathbf{A}_{22}\left(\mathbf{x}^{*}\right)^{\frac{1}{2}} \mathbf{e}_{\mathbf{v} \Omega}\right\|^{2}+c\left\|\mathbf{A}_{22}\left(\mathbf{x}^{*}\right)^{-\frac{1}{2}} \mathbf{M}_{22}\left(\mathbf{x}^{*}\right) \mathbf{d}_{\mathbf{v} \Omega}\right\|^{2} \\
& =\frac{1}{4}\left\|\mathbf{e}_{\mathbf{v} \Omega}\right\|_{\mathbf{A}_{22}\left(\mathbf{x}^{*}\right)}^{2}+c\left\|\mathbf{d}_{\mathbf{v} \Omega}\right\|_{\star, \mathbf{x}^{*}}^{2}
\end{aligned}
$$


The combination of the three estimates with absorptions (for $h \leqslant h_{0}$ sufficiently small) yields

$$
\left\|\dot{\mathbf{e}}_{\mathbf{x}^{\Omega}}\right\|_{\mathbf{A}_{22}\left(\mathbf{x}^{*}\right)}^{2}=\left\|\mathbf{e}_{\mathbf{v} \Omega}\right\|_{\mathbf{A}_{22}\left(\mathbf{x}^{*}\right)}^{2} \leqslant c\left\|\mathbf{e}_{\mathbf{x}^{\Omega}}\right\|_{\mathbf{A}_{22}\left(\mathbf{x}^{*}\right)}^{2}+c\left\|\mathbf{d}_{\mathbf{v}^{\Omega}}\right\|_{\star, \mathbf{x}^{*}}^{2} .
$$

We connect $\frac{d}{d t}\left\|\mathbf{e}_{\mathbf{x}^{\Omega}}\right\|_{\mathbf{A}_{22}\left(\mathbf{x}^{*}\right)}^{2}$ and $\left\|\dot{\mathbf{e}}_{\mathbf{x}^{\Omega}}\right\|_{\mathbf{A}_{22}\left(\mathbf{x}^{*}\right)}^{2}$. We have

$$
\frac{1}{2} \frac{\mathrm{d}}{\mathrm{d} t}\left\|\mathbf{e}_{\mathbf{x}^{\Omega}}\right\|_{\mathbf{A}_{22}\left(\mathbf{x}^{*}\right)}^{2}=\mathbf{e}_{\mathbf{x}^{\Omega}}^{\mathrm{T}} \mathbf{A}_{22}\left(\mathbf{x}^{*}\right) \dot{\mathbf{e}}_{\mathbf{x}^{\Omega}}+\frac{1}{2} \mathbf{e}_{\mathbf{x}^{\Omega}}^{\mathrm{T}}\left(\frac{\mathrm{d}}{\mathrm{d} t} \mathbf{A}_{22}\left(\mathbf{x}^{*}(t)\right)\right) \mathbf{e}_{\mathbf{x}^{\Omega}} .
$$

With the Cauchy-Schwarz and Young inequalities, we obtain

$$
\mathbf{e}_{\mathbf{x}^{\Omega}}^{\mathrm{T}} \mathbf{A}_{22}\left(\mathbf{x}^{*}\right) \dot{\mathbf{e}}_{\mathbf{x}^{\Omega}} \leqslant\left\|\mathbf{e}_{\mathbf{x}^{\Omega}}\right\|_{\mathbf{A}_{22}\left(\mathbf{x}^{*}\right)}\left\|\dot{\mathbf{e}}_{\mathbf{x}^{\Omega}}\right\|_{\mathbf{A}_{22}\left(\mathbf{x}^{*}\right)} \leqslant\left\|\dot{\mathbf{e}}_{\mathbf{x}^{\Omega}}\right\|_{\mathbf{A}_{22}\left(\mathbf{x}^{*}\right)}^{2}+\frac{1}{4}\left\|\mathbf{e}_{\mathbf{x}^{\Omega}}\right\|_{\mathbf{A}_{22}\left(\mathbf{x}^{*}\right)}^{2} .
$$

For the second term, Lemma 5.4 yields

$$
\frac{1}{2} \mathbf{e}_{\mathbf{x}^{\Omega}}^{\mathrm{T}}\left(\frac{\mathrm{d}}{\mathrm{d} t} \mathbf{A}_{22}\left(\mathbf{x}^{*}(t)\right)\right) \mathbf{e}_{\mathbf{x}^{\Omega}} \leqslant C\left\|\mathbf{e}_{\mathbf{x}^{\Omega}}(t)\right\|_{\mathbf{A}_{22}\left(\mathbf{x}^{*}(t)\right)}^{2} .
$$

We thus obtain, using (6.13)

$$
\frac{1}{2} \frac{\mathrm{d}}{\mathrm{d} t}\left\|\mathbf{e}_{\mathbf{x}^{\Omega}}\right\|_{\mathbf{A}_{22}\left(\mathbf{x}^{*}\right)}^{2} \leqslant c\left\|\mathbf{e}_{\mathbf{x}^{\Omega}}\right\|_{\mathbf{A}_{22}\left(\mathbf{x}^{*}\right)}^{2}+c\left\|\mathbf{d}_{\mathbf{v} \Omega}\right\|_{\star, \mathbf{x}^{*}}^{2} .
$$

Integrating from 0 to $t$ and using $\mathbf{e}_{\mathbf{x}^{\Omega}}(0)=0$, we obtain

$$
\left\|\mathbf{e}_{\mathbf{x}^{\Omega}}(t)\right\|_{\mathbf{A}_{22}\left(\mathbf{x}^{*}(t)\right)}^{2} \leqslant c \int_{0}^{t}\left\|\mathbf{d}_{\mathbf{v} \Omega}(s)\right\|_{*, \mathbf{x}^{*}(s)}^{2} \mathrm{~d} s+\int_{0}^{t} c\left\|\mathbf{e}_{\mathbf{x}^{\Omega}}(s)\right\|_{\mathbf{A}_{22}\left(\mathbf{x}^{*}(s)\right)}^{2} \mathrm{~d} s .
$$

The Gronwall inequality thus yields (6.7), which then inserted into (6.13) yields (6.8).

Now it remains to show that for $h \leqslant h_{0}$ sufficiently small we in fact have $t^{*}=T$. For $0 \leqslant t \leqslant t^{*}$, we have with an inverse inequality (see Brenner \& Scott (2007)) and for $h \leqslant h_{0}$ sufficiently small:

$$
\begin{aligned}
\left\|\nabla e_{x}(\cdot, t)\right\|_{L^{\infty}\left(\Omega_{h}\left(\mathbf{x}^{*}(t)\right)\right)} & \leqslant c h^{-3 / 2}\left\|\nabla e_{x}(\cdot, t)\right\|_{L^{2}\left(\Omega_{h}\left(\mathbf{x}^{*}(t)\right)\right)} \\
& =c h^{-3 / 2}\left\|\mathbf{e}_{\mathbf{x}^{\Omega}}(t)\right\|_{\mathbf{A}_{22}\left(\mathbf{x}^{*}(t)\right)} \leqslant c h^{\kappa-3 / 2} \\
& \leqslant \frac{1}{2} h^{\frac{\kappa-3 / 2}{2}} .
\end{aligned}
$$

This shows that the bound (6.9) can be extended beyond $t^{*}$, which contradicts the maximality of $t^{*}$ unless $t^{*}=T$.

REMARK 6.1 The previous lemma remains valid in the two-dimensional case, where the assumption (6.6) is only required for $\kappa>1$. Either way, it requires the finite element method to be of order two, at least.

\section{Stability of the semi-discrete diffusion equation}

In this section, we extend the stability result to the nodal vector $\mathbf{u}(t)$ of the numerical solution to the semi-discrete diffusion equation. 


\subsection{Error equations}

The numerical solution $u_{h}(x, t)=\sum_{j=1}^{N} u_{j}(t) \varphi_{j}[\mathbf{x}(t)](x)$ with corresponding nodal vector $\mathbf{u}=\mathbf{u}(t)=$ $\left(u_{j}(t)\right)_{j=1}^{N}$ satisfies

$$
\frac{\mathrm{d}}{\mathrm{d} t}(\mathbf{M}(\mathbf{x}) \mathbf{u})+\mathbf{A}(\mathbf{x}) \mathbf{u}=\mathbf{f}(\mathbf{x}) .
$$

The finite element interpolation $u_{h}^{*}(\cdot, t)$ of the exact solution $u(\cdot, t)$ with corresponding nodal vector $\mathbf{u}^{*}(t)$, when inserted into the matrix-vector formulation, yields defects $\mathbf{d}_{\mathbf{u}}$, corresponding to a finite element function $d_{h}^{u}$, such that

$$
\frac{\mathrm{d}}{\mathrm{d} t}\left(\mathbf{M}\left(\mathbf{x}^{*}\right) \mathbf{u}^{*}\right)+\mathbf{A}\left(\mathbf{x}^{*}\right) \mathbf{u}^{*}=\mathbf{f}\left(\mathbf{x}^{*}\right)+\mathbf{M}\left(\mathbf{x}^{*}\right) \mathbf{d}_{\mathbf{u}} .
$$

Rewriting (7.1) in a similar way as (6.3) and subtracting from (7.2) yields the error equation

$$
\begin{aligned}
\frac{\mathrm{d}}{\mathrm{d} t}\left(\mathbf{M}\left(\mathbf{x}^{*}\right) \mathbf{e}_{\mathbf{u}}\right)+\mathbf{A}\left(\mathbf{x}^{*}\right) \mathbf{e}_{\mathbf{u}}= & -\frac{\mathrm{d}}{\mathrm{d} t}\left(\left(\mathbf{M}(\mathbf{x})-\mathbf{M}\left(\mathbf{x}^{*}\right)\right) \mathbf{u}^{*}\right)-\frac{\mathrm{d}}{\mathrm{d} t}\left(\left(\mathbf{M}(\mathbf{x})-\mathbf{M}\left(\mathbf{x}^{*}\right)\right) \mathbf{e}_{\mathbf{u}}\right) \\
& -\left(\mathbf{A}(\mathbf{x})-\mathbf{A}\left(\mathbf{x}^{*}\right)\right) \mathbf{u}^{*}-\left(\mathbf{A}(\mathbf{x})-\mathbf{A}\left(\mathbf{x}^{*}\right)\right) \mathbf{e}_{\mathbf{u}}+\left(\mathbf{f}(\mathbf{x})-\mathbf{f}\left(\mathbf{x}^{*}\right)\right)-\mathbf{M}\left(\mathbf{x}^{*}\right) \mathbf{d}_{\mathbf{u}} .
\end{aligned}
$$

\subsection{Dual norm}

In order to bound the defect in $u$, we need to introduce a different dual norm than in the previous section, which is due to the fact that the defect $d_{h}^{u}$ lives on the whole domain $\Omega(t)$ and does not vanish on the boundary. We use the notation $\mathbf{K}\left(\mathbf{x}^{*}\right)=\mathbf{M}\left(\mathbf{x}^{*}\right)+\mathbf{A}\left(\mathbf{x}^{*}\right)$ and consider the dual norm (cf. (6.5))

$$
\begin{aligned}
\left\|d_{h}^{u}\right\|_{H_{h}^{-1}\left(\Omega_{h}\left(\mathbf{x}^{*}\right)\right)} & =\sup _{0 \neq \psi_{h} \in S_{h}\left(\mathbf{x}^{*}\right)} \frac{\int_{\Omega_{h}\left(\mathbf{x}^{*}\right)} d_{h}^{u} \psi_{h} \mathrm{~d} x}{\left\|\psi_{h}\right\|_{H^{1}\left(\Omega_{h}\left(\mathbf{x}^{*}\right)\right)}} \\
& =\left(\mathbf{d}_{\mathbf{u}}^{\mathrm{T}} \mathbf{M}\left(\mathbf{x}^{*}\right) \mathbf{K}\left(\mathbf{x}^{*}\right)^{-1} \mathbf{M}\left(\mathbf{x}^{*}\right) \mathbf{d}_{\mathbf{u}}\right)^{1 / 2}=:\left\|\mathbf{d}_{\mathbf{u}}\right\|_{\star, \mathbf{x}} \mathbf{x}^{*} .
\end{aligned}
$$

For simplicity, we do not use another notation for the dual norm of $\mathbf{d}_{\mathbf{u}}$, as it will always be clear from context which dual norm is meant. In the following stability proof, we need the following technical lemma.

LEMMA 7.1 For a function $w=w(x, t): \Omega(t) \rightarrow \mathbb{R}^{3}$, we have

$$
\partial^{\bullet}(\nabla \cdot w)=\nabla \cdot \partial \bullet w-\nabla v \cdot \nabla w,
$$

where $v=v(x, t)$ is the velocity and $\nabla v \cdot \nabla w$ denotes the Frobenius norm inner product, i.e. the Euclidean product of the vectorizations of the matrices.

Proof. Based on Dziuk et al. (2013), a similar identity for the surface divergence is shown in Kovács et al. (2017). The proof is adapted by embedding everything into a surface $\Gamma(t)=\Omega(t) \times\{0\} \in \mathbb{R}^{4}$.

\subsection{Stability estimate}

We are now able to state and prove the stability result for the error $\mathbf{e}_{\mathbf{u}}$. Note that the previous stability estimates for $\mathbf{e}_{\mathbf{x}}$ and $\mathbf{e}_{\mathbf{v}}$ remain valid since the solution to the domain evolution does not depend on the numerical solution $u_{h}$, but the solution $u_{h}$ to the diffusion equation depends on the solution $\mathbf{x}$ of the position vectors, which is reflected in the following proof. 
LEMMA 7.2 Assume that, for some $\kappa>\frac{3}{2}$, the defects are bounded as follows:

$$
\left\|\mathbf{d}_{\mathbf{u}}(t)\right\|_{\star, \mathbf{x}^{*}(t)} \leqslant c h^{\kappa}, \quad\left\|\mathbf{d}_{\mathbf{v}^{\Omega}}(t)\right\|_{\star, \mathbf{x}^{*}(t)} \leqslant c h^{\kappa}, \quad t \in[0, T] .
$$

Then there exists an $h_{0}>0$ such that the following estimate holds for $h \leqslant h_{0}$ and $t \in[0, T]$, where the constant $C$ is independent of $h$ :

$$
\left\|\mathbf{e}_{\mathbf{u}}(t)\right\|_{\mathbf{M}\left(\mathbf{x}^{*}\right)}^{2}+\int_{0}^{t}\left\|\mathbf{e}_{\mathbf{u}}(s)\right\|_{\mathbf{A}\left(\mathbf{x}^{*}(s)\right)}^{2} \mathrm{~d} s \leqslant C \int_{0}^{t}\left\|\mathbf{d}_{\mathbf{u}}(s)\right\|_{\star, \mathbf{x}^{*}(s)}^{2}+\left\|\mathbf{d}_{\mathbf{v}}(s)\right\|_{\star, \mathbf{x}^{*}(s)}^{2} \mathrm{~d} s .
$$

Proof. The proof is similar to the proof of Lemma 6.1. Let $0<t^{*} \leqslant T$ be the maximal time such that

$$
\begin{aligned}
\left\|\nabla e_{x}(\cdot, t)\right\|_{L^{\infty}\left(\Omega_{h}\left(\mathbf{x}^{*}(t)\right)\right)} & \leqslant h^{(\kappa-3 / 2) / 2}, \\
\left\|e_{u}(\cdot, t)\right\|_{L^{\infty}\left(\Omega_{h}\left(\mathbf{x}^{*}(t)\right)\right)} & \leqslant 1 .
\end{aligned}
$$

for all $t \in\left[0, t^{*}\right]$. Note that $e_{x}(\cdot, 0)=0=e_{u}(\cdot, 0)$ implies $t^{*}>0$. Again, we will prove the error bound for $t \in\left[0, t^{*}\right]$ and then show that $t^{*}$ coincides with $T$.

Testing (7.3) with $\mathbf{e}_{\mathbf{u}}^{\mathrm{T}}$, we obtain (omitting the argument $t$ )

$$
\begin{aligned}
\mathbf{e}_{\mathbf{u}}^{\mathrm{T}} \frac{\mathrm{d}}{\mathrm{d} t}\left(\mathbf{M}\left(\mathbf{x}^{*}\right) \mathbf{e}_{\mathbf{u}}\right)+\mathbf{e}_{\mathbf{u}}^{\mathrm{T}} \mathbf{A}\left(\mathbf{x}^{*}\right) \mathbf{e}_{\mathbf{u}}= & -\mathbf{e}_{\mathbf{u}}^{\mathrm{T}} \frac{\mathrm{d}}{\mathrm{d} t}\left(\left(\mathbf{M}(\mathbf{x})-\mathbf{M}\left(\mathbf{x}^{*}\right)\right) \mathbf{u}^{*}\right)-\mathbf{e}_{\mathbf{u}}^{\mathrm{T}} \frac{\mathrm{d}}{\mathrm{d} t}\left(\left(\mathbf{M}(\mathbf{x})-\mathbf{M}\left(\mathbf{x}^{*}\right)\right) \mathbf{e}_{\mathbf{u}}\right) \\
& -\mathbf{e}_{\mathbf{u}}^{\mathrm{T}}\left(\mathbf{A}(\mathbf{x})-\mathbf{A}\left(\mathbf{x}^{*}\right)\right) \mathbf{u}^{*}-\mathbf{e}_{\mathbf{u}}^{\mathrm{T}}\left(\mathbf{A}(\mathbf{x})-\mathbf{A}\left(\mathbf{x}^{*}\right)\right) \mathbf{e}_{\mathbf{u}} \\
& -\mathbf{e}_{\mathbf{u}}^{\mathrm{T}}\left(\mathbf{f}(\mathbf{x})-\mathbf{f}\left(\mathbf{x}^{*}\right)\right)-\mathbf{e}_{\mathbf{u}}^{\mathrm{T}} \mathbf{M}\left(\mathbf{x}^{*}\right) \mathbf{d}_{\mathbf{u}}
\end{aligned}
$$

We estimate the six terms on the right-hand side separately.

(i) We apply the product rule to obtain

$$
\mathbf{e}_{\mathbf{u}}^{\mathrm{T}} \frac{\mathrm{d}}{\mathrm{d} t}\left(\left(\mathbf{M}(\mathbf{x})-\mathbf{M}\left(\mathbf{x}^{*}\right)\right) \mathbf{u}^{*}\right)=\mathbf{e}_{\mathbf{u}}^{\mathrm{T}}\left(\mathbf{M}(\mathbf{x})-\mathbf{M}\left(\mathbf{x}^{*}\right)\right) \dot{\mathbf{u}}^{*}+\mathbf{e}_{\mathbf{u}}^{\mathrm{T}}\left(\frac{\mathrm{d}}{\mathrm{d} t}\left(\mathbf{M}(\mathbf{x})-\mathbf{M}\left(\mathbf{x}^{*}\right)\right)\right) \mathbf{u}^{*} .
$$

For the first term of (7.7), we use Lemma 5.1, an $L^{2}-L^{2}-L^{\infty}$-estimate and Lemma 5.3 to obtain

$$
\begin{aligned}
\left|\mathbf{e}_{\mathbf{u}}^{\mathrm{T}}\left(\mathbf{M}(\mathbf{x})-\mathbf{M}\left(\mathbf{x}^{*}\right)\right) \dot{\mathbf{u}}^{*}\right| & =\left|\int_{0}^{1} \int_{\Omega_{h}^{\theta}} e_{u}^{\theta}\left(\nabla \cdot e_{x}^{\theta}\right) \partial_{h}^{\bullet} u_{h}^{*, \theta} \mathrm{d} x \mathrm{~d} \theta\right| \\
& \leqslant\left.\int_{0}^{1}\left\|e_{u}^{\theta}\right\|_{L^{2}\left(\Omega_{h}^{\theta}\right)}\left\|\nabla \cdot e_{x}^{\theta}\right\|\right|_{L^{2}\left(\Omega_{h}^{\theta}\right)}\left\|\partial_{h}^{\bullet} u_{h}^{*, \theta}\right\|_{L^{\infty}\left(\Omega_{h}^{\theta}\right)} \mathrm{d} \theta \\
& \leqslant c\left\|\mathbf{e}_{\mathbf{u}}\right\|_{\mathbf{M}\left(\mathbf{x}^{*}\right)}\left\|\mathbf{e}_{\mathbf{x}}\right\|_{\mathbf{A}\left(\mathbf{x}^{*}\right)}\left\|\partial_{h}^{\bullet} u_{h}^{*, 0}\right\| \|_{L^{\infty}\left(\Omega_{h}\left(\mathbf{x}^{*}\right)\right)} .
\end{aligned}
$$

With an elementary computation, the last term can be bounded by $\left\|\partial_{h}^{\bullet} u_{h}^{*, 0}\right\|_{L^{\infty}\left(\Omega_{h}\left(\mathbf{x}^{*}\right)\right)} \leqslant c\left\|\dot{\mathbf{u}}^{*}(t)\right\|_{\infty}$, and the nodal values of $\dot{\mathbf{u}}^{*}(t)$ are exactly the nodal values of $\partial^{\bullet} u(\cdot, t)$. The smoothness assumption on $u$ and $\partial^{\bullet} u$ thus implies $\left\|\dot{\mathbf{u}}^{*}\right\|_{\infty} \leqslant c$, and we arrive at

$$
\left|\mathbf{e}_{\mathbf{u}}^{\mathrm{T}}\left(\mathbf{M}(\mathbf{x})-\mathbf{M}\left(\mathbf{x}^{*}\right)\right) \dot{\mathbf{u}}^{*}\right| \leqslant c\left\|\mathbf{e}_{\mathbf{u}}\right\|_{\mathbf{M}\left(\mathbf{x}^{*}\right)}\left\|\mathbf{e}_{\mathbf{x}}\right\|_{\mathbf{A}\left(\mathbf{x}^{*}\right)} \cdot
$$

Using the basis functions, Lemma 5.1 and the Leibniz formula, a tedious but elementary computation yields

$$
\begin{aligned}
\mathbf{e}_{\mathbf{u}}^{\mathrm{T}} \frac{\mathrm{d}}{\mathrm{d} t}\left(\mathbf{M}(\mathbf{x})-\mathbf{M}\left(\mathbf{x}^{*}\right)\right) \mathbf{u}^{*} & =\int_{0}^{1} \int_{\Omega_{h}^{\theta}} e_{u}^{\theta} \partial_{h}^{\bullet} \nabla \cdot e_{x}^{\theta} u_{h}^{*, \theta} \mathrm{d} x \mathrm{~d} \theta \\
& +\int_{0}^{1} \int_{\Omega_{h}^{\theta}} e_{u}^{\theta}\left(\nabla \cdot e_{x}^{\theta}\right) u_{h}^{*, \theta} \nabla \cdot v_{h}^{\theta} \mathrm{d} x \mathrm{~d} \theta
\end{aligned}
$$


where $v_{h}^{\theta}$ is the velocity of $\Omega_{h}^{\theta}$ as a function of $t$, i. e. the finite element function in $S_{h}\left(\mathbf{x}^{*}(t)+\theta \mathbf{e}_{\mathbf{x}}(t)\right)$ with nodal vector $\dot{\mathbf{x}}^{*}+\theta \dot{\mathbf{e}}_{\mathbf{x}}=\mathbf{v}^{*}+\theta \mathbf{e}_{\mathbf{v}}$, implying $v_{h}^{\theta}=v_{h}^{*, \theta}+\theta e_{v}^{\theta}$. We will estimate both integrals separately, where we use the identity from Lemma 7.1. With $\partial_{h}^{\bullet} e_{x}^{\theta}=e_{v}^{\theta}$ and writing $v_{h}^{\theta}=v_{h}^{*, \theta}+\theta e_{v}^{\theta}$, we obtain the following estimate for the first integral: (we write $L^{p}$ instead of $L^{p}\left(\Omega_{h}^{\theta}\right)$ and $\mathbf{M}$ and $\mathbf{A}$ instead of $\mathbf{M}\left(\mathbf{x}^{*}\right)$ and $\mathbf{A}\left(\mathbf{x}^{*}\right)$ in the occurring norms)

$$
\begin{aligned}
& \left|\int_{0}^{1} \int_{\Omega_{h}^{\theta}} e_{u}^{\theta} \partial_{h}^{\bullet} \nabla \cdot e_{x}^{\theta} u_{h}^{*, \theta} \mathrm{d} x \mathrm{~d} \theta\right| \\
& \leqslant \int_{0}^{1}\left\|e_{u}^{\theta}\right\|_{L^{2}}\left(\left\|\nabla \cdot e_{v}^{\theta}\right\|_{L^{2}}+\left\|\nabla v_{h}^{*, \theta}\right\|_{L^{\infty}}\left\|\nabla e_{x}^{\theta}\right\|_{L^{2}}+\theta\left\|\nabla e_{v}^{\theta}\right\|_{L^{2}}\left\|\nabla e_{x}^{\theta}\right\|_{L^{\infty}}\right)\left\|u_{h}^{*, \theta}\right\|_{L^{\infty}} \\
& \leqslant c\left\|\mathbf{e}_{\mathbf{u}}\right\|_{L^{2}}\left(\left\|\nabla e_{v}\right\|_{L^{2}}+\left\|\nabla v_{h}^{*}\right\|_{L^{\infty}}\left\|\nabla e_{x}\right\|_{L^{2}}+\left\|\nabla e_{v}\right\|_{L^{2}}\left\|\nabla e_{x}\right\|_{\left.L^{\infty}\right)}\left\|u_{h}^{*}\right\|_{L^{\infty}}\right. \\
& \leqslant c\left\|\mathbf{e}_{\mathbf{u}}\right\|_{\mathbf{M}}\left(\left\|\mathbf{e}_{\mathbf{v}}\right\|_{\mathbf{A}}+\left\|\nabla v_{h}^{*}\right\|_{L^{\infty}}\left\|\mathbf{e}_{\mathbf{x}}\right\|_{\mathbf{A}}+\left\|\mathbf{e}_{\mathbf{v}}\right\|_{\mathbf{A}}\left\|\nabla e_{x}\right\|_{L^{\infty}}\right)\left\|\mathbf{u}^{*}\right\|_{\infty} \\
& \leqslant c\left\|\mathbf{e}_{\mathbf{u}}\right\|_{\mathbf{M}\left(\mathbf{x}^{*}\right)}\left(\left\|\mathbf{e}_{\mathbf{v}}\right\|_{\mathbf{A}\left(\mathbf{x}^{*}\right)}+\left\|\mathbf{e}_{\mathbf{x}}\right\|_{\mathbf{A}\left(\mathbf{x}^{*}\right)}\right) .
\end{aligned}
$$

We analogously estimate the second integral and obtain

$$
\left|\int_{0}^{1} \int_{\Omega_{h}^{\theta}} e_{u}^{\theta}\left(\nabla \cdot e_{x}^{\theta}\right) u_{h}^{*, \theta} \nabla \cdot v_{h}^{\theta} \mathrm{d} x \mathrm{~d} \theta\right| \leqslant c\left\|\mathbf{e}_{\mathbf{u}}\right\|_{\mathbf{M}\left(\mathbf{x}^{*}\right)}\left(\left\|\mathbf{e}_{\mathbf{v}}\right\|_{\mathbf{A}\left(\mathbf{x}^{*}\right)}+\left\|\mathbf{e}_{\mathbf{x}}\right\|_{\mathbf{A}\left(\mathbf{x}^{*}\right)}\right) .
$$

Finally, we obtain for the first term of (7.6):

$$
-\mathbf{e}_{\mathbf{u}}^{\mathrm{T}} \frac{\mathrm{d}}{\mathrm{d} t}\left(\left(\mathbf{M}(\mathbf{x})-\mathbf{M}\left(\mathbf{x}^{*}\right)\right) \mathbf{u}^{*}\right) \leqslant c\left\|\mathbf{e}_{\mathbf{u}}\right\|_{\mathbf{M}\left(\mathbf{x}^{*}\right)}\left(\left\|\mathbf{e}_{\mathbf{v}}\right\|_{\mathbf{A}\left(\mathbf{x}^{*}\right)}+\left\|\mathbf{e}_{\mathbf{x}}\right\|_{\mathbf{A}\left(\mathbf{x}^{*}\right)}\right) .
$$

(ii) For the second term of (7.6), we obtain similarly

$$
\begin{aligned}
& -\mathbf{e}_{\mathbf{u}}^{\mathrm{T}} \frac{\mathrm{d}}{\mathrm{d} t}\left(\left(\mathbf{M}(\mathbf{x})-\mathbf{M}\left(\mathbf{x}^{*}\right)\right) \mathbf{e}_{\mathbf{u}}\right) \\
& =-\frac{1}{2} \mathbf{e}_{\mathbf{u}}^{\mathrm{T}}\left(\frac{\mathrm{d}}{\mathrm{d} t}\left(\mathbf{M}(\mathbf{x})-\mathbf{M}\left(\mathbf{x}^{*}\right)\right)\right) \mathbf{e}_{\mathbf{u}}-\frac{1}{2} \frac{\mathrm{d}}{\mathrm{d} t}\left(\mathbf{e}_{\mathbf{u}}^{\mathrm{T}}\left(\mathbf{M}(\mathbf{x})-\mathbf{M}\left(\mathbf{x}^{*}\right) \mathbf{e}_{\mathbf{u}}\right)\right. \\
& \leqslant c\left\|\mathbf{e}_{\mathbf{u}}\right\|_{\mathbf{M}\left(\mathbf{x}^{*}\right)}\left(\left\|\mathbf{e}_{\mathbf{v}}\right\|_{\mathbf{A}\left(\mathbf{x}^{*}\right)}+\left\|\mathbf{e}_{\mathbf{x}}\right\|_{\mathbf{A}\left(\mathbf{x}^{*}\right)}\right)\left\|e_{u}\right\|_{L^{\infty}\left(\Omega_{h}\left(\mathbf{x}^{*}\right)\right)}-\frac{1}{2} \frac{\mathrm{d}}{\mathrm{d} t}\left(\mathbf{e}_{\mathbf{u}}^{\mathrm{T}}\left(\mathbf{M}(\mathbf{x})-\mathbf{M}\left(\mathbf{x}^{*}\right)\right) \mathbf{e}_{\mathbf{u}}\right) \\
& \leqslant C\left\|\mathbf{e}_{\mathbf{u}}\right\|_{\mathbf{M}\left(\mathbf{x}^{*}\right)}\left(\left\|\mathbf{e}_{\mathbf{v}}\right\|_{\mathbf{A}\left(\mathbf{x}^{*}\right)}+\left\|\mathbf{e}_{\mathbf{x}}\right\|_{\mathbf{A}\left(\mathbf{x}^{*}\right)}\right)-\frac{1}{2} \frac{\mathrm{d}}{\mathrm{d} t}\left(\mathbf{e}_{\mathbf{u}}^{\mathrm{T}}\left(\mathbf{M}(\mathbf{x})-\mathbf{M}\left(\mathbf{x}^{*}\right)\right) \mathbf{e}_{\mathbf{u}}\right) .
\end{aligned}
$$

(iii) For the third term, we use Lemma 5.1 and Lemma 5.3 and estimate

$$
\begin{aligned}
\left|\mathbf{e}_{\mathbf{u}}^{\mathrm{T}}\left(\mathbf{A}(\mathbf{x})-\mathbf{A}\left(\mathbf{x}^{*}\right)\right) \mathbf{u}^{*}\right| & \leqslant c \int_{0}^{1}\left\|\nabla e_{u}^{\theta}\right\|_{L^{2}\left(\Omega_{h}^{\theta}\right)}\left\|\nabla e_{x}^{\theta}\right\|_{L^{2}\left(\Omega_{h}^{\theta}\right)}\left\|\nabla u_{h}^{*, \theta}\right\|_{L^{\infty}\left(\Omega_{h}^{\theta}\right)} \mathrm{d} x \mathrm{~d} \theta \\
& \leqslant c\left\|\nabla e_{u}\right\|_{L^{2}\left(\Omega_{h}\left(\mathbf{x}^{*}\right)\right)}\left\|\nabla e_{x}\right\|_{L^{2}\left(\Omega_{h}\left(\mathbf{x}^{*}\right)\right)}\left\|\nabla u_{h}^{*}\right\|_{L^{\infty}\left(\Omega_{h}\left(\mathbf{x}^{*}\right)\right)} \\
& \leqslant C\left\|\mathbf{e}_{\mathbf{u}}\right\|_{\mathbf{A}\left(\mathbf{x}^{*}\right)}\left\|\mathbf{e}_{\mathbf{x}}\right\|_{\mathbf{A}\left(\mathbf{x}^{*}\right)},
\end{aligned}
$$

where we have used the smoothness assumption on $u$.

(iv) Similarly, we estimate

$$
\begin{aligned}
\left|\mathbf{e}_{\mathbf{u}}^{\mathrm{T}}\left(\mathbf{A}(\mathbf{x})-\mathbf{A}\left(\mathbf{x}^{*}\right)\right) \mathbf{e}_{\mathbf{u}}\right| & =\left|\int_{0}^{1} \int_{\Omega_{h}^{\theta}} \nabla e_{u}^{\theta}\left(D_{\Omega_{h}^{\theta}} e_{x}^{\theta}\right) \nabla e_{u}^{\theta} \mathrm{d} x \mathrm{~d} \theta\right| \\
& \leqslant c\left\|\nabla e_{u}\right\|_{L^{2}\left(\Omega_{h}\left(\mathbf{x}^{*}\right)\right)}^{2}\left\|\nabla e_{x}\right\|_{L^{\infty}\left(\Omega_{h}\left(\mathbf{x}^{*}\right)\right)} \\
& \leqslant c h^{(\kappa-3 / 2) / 2}\left\|\mathbf{e}_{\mathbf{u}}\right\|_{\mathbf{A}\left(\mathbf{x}^{*}\right)}^{2}
\end{aligned}
$$


(v) For the fifth term, we use the Leibniz formula, an $L^{\infty}-L^{2}-L^{2}$-estimate and Lemma 5.3 to obtain

$$
\begin{aligned}
\mathbf{e}_{\mathbf{u}}^{\mathrm{T}}\left(\mathbf{f}(\mathbf{x})-\mathbf{f}\left(\mathbf{x}^{*}\right)\right) & =\int_{\Omega_{h}^{1}} f e_{u}^{1} \mathrm{~d} x-\int_{\Omega_{h}^{0}} f e_{u}^{\theta} \mathrm{d} x=\int_{0}^{1} \frac{\mathrm{d}}{\mathrm{d} \theta} \int_{\Omega_{h}^{\theta}} f e_{u}^{\theta} \mathrm{d} x \mathrm{~d} \theta \\
& =\int_{0}^{1} \int_{\Omega_{h}^{\theta}} \partial_{\theta}^{\bullet} f e_{u}^{\theta}+f \underbrace{\partial_{\theta}^{\bullet} e_{u}^{\theta}}_{=0}+f e_{u}^{\theta} \nabla \cdot e_{x}^{\theta} \mathrm{d} x \mathrm{~d} \theta \\
& =\int_{0}^{1} \int_{\Omega_{h}^{\theta}} f^{\prime} e_{x}^{\theta} e_{u}^{\theta}+f e_{u}^{\theta} \nabla \cdot e_{x}^{\theta} \mathrm{d} x \mathrm{~d} \theta \\
& \leqslant \int_{0}^{1}\left\|f^{\prime}\right\|_{L^{\infty}\left(\Omega_{h}^{\theta}\right)}\left\|e_{x}^{\theta}\right\|_{L^{2}\left(\Omega_{h}^{\theta}\right)}\left\|e_{u}^{\theta}\right\|_{L^{2}\left(\Omega_{h}^{\theta}\right)}+\|f\|_{L^{\infty}\left(\Omega_{h}^{\theta}\right)}\left\|e_{u}^{\theta}\right\|_{L^{2}\left(\Omega_{h}^{\theta}\right)}\left\|\nabla \cdot e_{x}^{\theta}\right\|_{L^{2}\left(\Omega_{h}^{\theta}\right)} \mathrm{d} \theta \\
& \leqslant c\left\|\mathbf{e}_{\mathbf{x}}\right\|_{\mathbf{M}\left(\mathbf{x}^{*}\right)}\left\|\mathbf{e}_{\mathbf{u}}\right\|_{\mathbf{M}\left(\mathbf{x}^{*}\right)}+c\left\|\mathbf{e}_{\mathbf{x}}\right\|_{\mathbf{A}\left(\mathbf{x}^{*}\right)}\left\|\mathbf{e}_{\mathbf{u}}\right\|_{\mathbf{M}\left(\mathbf{x}^{*}\right)} \\
& \leqslant c\left\|\mathbf{e}_{\mathbf{x}}\right\|_{\mathbf{A}\left(\mathbf{x}^{*}\right)}\left\|\mathbf{e}_{\mathbf{u}}\right\|_{\mathbf{M}\left(\mathbf{x}^{*}\right)},
\end{aligned}
$$

where we have used the Poincaré inequality in the last step, which yields for $e_{x}^{\ell} \in H_{0}^{1}(\Omega(t))$

$$
\left\|\mathbf{e}_{\mathbf{x}}\right\|_{\mathbf{M}\left(\mathbf{x}^{*}\right)}=\left\|e_{x}\right\|_{L^{2}\left(\Omega_{h}\left(\mathbf{x}^{*}(t)\right)\right)} \leqslant c\left\|e_{x}^{\ell}\right\|_{L^{2}(\Omega(t))} \leqslant c\left\|\nabla e_{x}^{\ell}\right\|_{L^{2}(\Omega(t))} \leqslant c\left\|\mathbf{e}_{\mathbf{x}}\right\|_{\mathbf{A}\left(\mathbf{x}^{*}\right)} .
$$

(vi) For the last term of (7.6), we use

$$
\begin{aligned}
\mathbf{e}_{\mathbf{u}}^{\mathrm{T}} \mathbf{M}\left(\mathbf{x}^{*}\right) \mathbf{d}_{\mathbf{u}} & =\mathbf{e}_{\mathbf{u}}^{\mathrm{T}} \mathbf{K}\left(\mathbf{x}^{*}\right)^{\frac{1}{2}} \mathbf{K}\left(\mathbf{x}^{*}\right)^{-\frac{1}{2}} \mathbf{M}\left(\mathbf{x}^{*}\right) \mathbf{d}_{\mathbf{u}} \\
& \leqslant \frac{1}{6}\left\|\mathbf{K}\left(\mathbf{x}^{*}\right)^{\frac{1}{2}} \mathbf{e}_{\mathbf{u}}\right\|_{2}^{2}+C\left\|\mathbf{K}\left(\mathbf{x}^{*}\right)^{-\frac{1}{2}} \mathbf{M}\left(\mathbf{x}^{*}\right) \mathbf{d}_{\mathbf{u}}\right\|_{2}^{2} \\
& =\frac{1}{6}\left\|\mathbf{e}_{\mathbf{u}}\right\|_{\mathbf{M}\left(\mathbf{x}^{*}\right)}^{2}+\frac{1}{6}\left\|\mathbf{e}_{\mathbf{u}}\right\|_{\mathbf{A}\left(\mathbf{x}^{*}\right)}^{2}+C\left\|\mathbf{d}_{\mathbf{u}}\right\|_{\star, \mathbf{x}^{*}}^{2}
\end{aligned}
$$

Combining estimates (i)-(vi), using Young's inequality on each product, for $h \leqslant h_{0}$ sufficiently small such that $c h^{(\kappa-3 / 2) / 2} \leqslant 1 / 6$, we obtain after absorbing $\left\|\mathbf{e}_{\mathbf{u}}\right\|_{\mathbf{A}\left(\mathbf{x}^{*}\right)}^{2}$ :

$$
\begin{aligned}
\frac{1}{2} \frac{\mathrm{d}}{\mathrm{d} t}\left\|\mathbf{e}_{\mathbf{u}}\right\|_{\mathbf{M}\left(\mathbf{x}^{*}\right)}^{2}+\frac{1}{2}\left\|\mathbf{e}_{\mathbf{u}}\right\|_{\mathbf{A}\left(\mathbf{x}^{*}\right)}^{2} & \leqslant c\left\|\mathbf{e}_{\mathbf{u}}\right\|_{\mathbf{M}\left(\mathbf{x}^{*}\right)}^{2}+c\left\|\mathbf{e}_{\mathbf{x}}\right\|_{\mathbf{A}\left(\mathbf{x}^{*}\right)}^{2}+c\left\|\mathbf{e}_{\mathbf{v}}\right\|_{\mathbf{A}\left(\mathbf{x}^{*}\right)}^{2} \\
& -\frac{1}{2} \frac{\mathrm{d}}{\mathrm{d} t}\left(\mathbf{e}_{\mathbf{u}}^{\mathrm{T}}\left(\mathbf{M}(\mathbf{x})-\mathbf{M}\left(\mathbf{x}^{*}\right)\right) \mathbf{e}_{\mathbf{u}}\right)+c\left\|\mathbf{d}_{\mathbf{u}}\right\|_{\star, \mathbf{x}^{*}}^{2} .
\end{aligned}
$$

Inserting the estimates from Lemma 6.1, we have

$$
\begin{aligned}
\frac{\mathrm{d}}{\mathrm{d} t}\left\|\mathbf{e}_{\mathbf{u}}\right\|_{\mathbf{M}\left(\mathbf{x}^{*}\right)}^{2}+\left\|\mathbf{e}_{\mathbf{u}}\right\|_{\mathbf{A}\left(\mathbf{x}^{*}\right)}^{2} & \leqslant c\left\|\mathbf{e}_{\mathbf{u}}\right\|_{\mathbf{M}\left(\mathbf{x}^{*}\right)}^{2}+c\left\|\mathbf{d}_{\mathbf{v}}\right\|_{\star, \mathbf{x}^{*}}^{2}+c \int_{0}^{t}\left\|\mathbf{d}_{\mathbf{v}}(s)\right\|_{\star, \mathbf{x}^{*}(s)}^{2} \mathrm{~d} s \\
& -\frac{\mathrm{d}}{\mathrm{d} t}\left(\mathbf{e}_{\mathbf{u}}^{\mathrm{T}}\left(\mathbf{M}(\mathbf{x})-\mathbf{M}\left(\mathbf{x}^{*}\right)\right) \mathbf{e}_{\mathbf{u}}\right)+c\left\|\mathbf{d}_{\mathbf{u}}\right\|_{\star, \mathbf{x}^{*}}^{2} .
\end{aligned}
$$

Integrating from 0 to $t$ for $t \in\left[0, t^{*}\right]$ and using a Gronwall argument as in part (C) of (Kovács et al., 2017, Proposition 6.1), we finally obtain the desired result for $t \in\left[0, t^{*}\right]$. The proof is then finished by showing that $t^{*}$ coincides with $T$, which is due to the same argument as in the previous section.

REMARK 7.1 The previous lemma remains valid in the two-dimensional case, where the assumption (7.5) is only required for $\kappa>1$. 


\section{Defect bounds}

In this section we show that the smallness assumptions in Lemma 6.1 and Lemma 7.2 are satisfied for $\kappa=k \geqslant 2$, which in combination with the stability results will lead to the desired error bounds. We remind that we have different dual norm definitions (6.5) and (7.4) since the defect functions live in different finite element spaces. We avoid using different notations, because the dual norms only appear on $\mathbf{d}_{\mathbf{v}}$ and $\mathbf{d}_{\mathbf{u}}$, so it is always clear from context which definition is meant.

\subsection{The interpolating domain}

In order to estimate the defect $\mathbf{d}_{\mathbf{u}}$, we need to introduce a discrete velocity on the smooth domain, which is denoted by $\widehat{v}_{h}$.

Recall that $\Omega(t)$ can be described as image $X(\cdot, t)\left(\Omega_{0}\right)$ with a sufficiently smooth map $X: \Omega_{0} \times$ $[0, T] \rightarrow \mathbb{R}^{3}$. The nodes $x_{j}^{*}(t)=X\left(x_{j}^{0}, t\right)$ define an interpolating domain which is parametrized over $\Omega_{h}^{0}$ via

$$
X_{h}^{*}\left(p_{h}, t\right)=\sum_{j=1}^{N} x_{j}^{*}(t) \varphi_{j}[\mathbf{x}(0)]\left(p_{h}\right), \quad p_{h} \in \Omega_{h}^{0}
$$

The velocity of the interpolating domain is given, using the transport property of the basis functions (3.1), by

$$
v_{h}^{*}(\cdot, t)=\sum_{j=1}^{N} v_{j}^{*}(t) \varphi_{j}\left[\mathbf{x}^{*}(t)\right](\cdot) \quad \text { with } v_{j}^{*}(t)=\frac{\mathrm{d}}{\mathrm{d} t} x_{j}^{*}(t) .
$$

For a material point $p_{h}(t)=X_{h}^{*}\left(p_{h}, t\right) \in \Omega_{h}\left(\mathbf{x}^{*}(t)\right), p_{h} \in \Omega_{h}^{0}$, on the interpolated exact domain, this velocity satisfies

$$
v_{h}^{*}\left(p_{h}(t), t\right)=\frac{\mathrm{d}}{\mathrm{d} t} X_{h}^{*}\left(p_{h}, t\right) .
$$

Associated with $p_{h}(t)$ is its lifted material point $y(t)=\Lambda_{h}\left(p_{h}(t), t\right) \in \Omega(t)$. This lifted point moves with velocity

$$
\widehat{v}_{h}(y(t), t)=\frac{\mathrm{d}}{\mathrm{d} t} y(t)=\frac{\mathrm{d}}{\mathrm{d} t} \Lambda_{h}\left(p_{h}(t), t\right)=\left(\partial_{t} \Lambda_{h}\right)\left(p_{h}(t), t\right)+v_{h}^{*}\left(p_{h}(t), t\right) \nabla \Lambda_{h}\left(p_{h}(t), t\right) .
$$

We can use these velocities to define discrete material derivatives for functions $\varphi_{h}$ and $\varphi$ defined on $\Omega_{h}\left(\mathbf{x}^{*}(t)\right)$ and $\Omega(t)$, respectively, via

$$
\begin{gathered}
\partial_{v_{h}^{*}}^{\bullet} \varphi_{h}=\partial_{t} \varphi_{h}+v_{h}^{*} \cdot \nabla \varphi_{h}, \\
\partial_{\hat{v}_{h}}^{\bullet} \varphi=\partial_{t} \varphi+\widehat{v}_{h} \cdot \nabla \varphi .
\end{gathered}
$$

The basis functions $\varphi_{j}\left[\mathbf{x}^{*}\right]$ enjoy the transport property $\partial_{v_{h}^{*}}^{\bullet} \varphi_{j}=0$. It is not true in general that the lifted basis functions satisfy $\partial_{v}^{\bullet} \varphi_{j}^{\ell}=0$, with $\partial_{v}^{\bullet}=\partial^{\bullet}$ as defined in (2.1). In particular, we have $\left(\partial_{v_{h}^{\bullet}}^{\bullet} \varphi_{j}\right)^{\ell} \neq \partial^{\bullet} \varphi_{j}^{\ell}$ in general. The following lemma shows that the transport property is satisfied with the discrete velocity defined above, which will be crucial in the following.

LEMMA 8.1 For $j=1, \ldots, N$, we have

$$
\partial_{\hat{v}_{h}}^{\bullet} \varphi_{j}^{\ell}=0 .
$$

In particular, we have for any finite element function $\eta_{h} \in S_{h}\left(\mathbf{x}^{*}(t)\right)$ and for any $u \in H^{k+1}(\Omega(t))$

$$
\left(\partial_{v_{h}^{*}}^{\bullet} \eta_{h}\right)^{\ell}=\partial_{\hat{v}_{h}}^{\bullet} \eta_{h}^{\ell} \quad \text { and } \quad\left(\partial_{v_{h}^{*}}^{\bullet} \widetilde{I}_{h} u\right)^{\ell}=\partial_{\widehat{v}_{h}}^{\bullet} I_{h} u=I_{h} \partial_{\hat{v}_{h}}^{\bullet} u
$$


Proof. Follows from Definition 3.1, the chain rule and the transport property of the basis functions, cf. (Dziuk \& Elliott, 2013, Lemma 4.1).

For the following defect estimate, we introduce the notation

$$
\begin{aligned}
q_{h}^{*}\left(\eta_{h}, \chi_{h}\right) & =\int_{\Omega_{h}^{*}(t)} \eta_{h} \chi_{h} \nabla \cdot v_{h}^{*} \mathrm{~d} x, \\
\widehat{q}_{h}(\eta, \chi) & =\int_{\Omega(t)} \eta \chi \nabla \cdot \widehat{v}_{h} \mathrm{~d} x .
\end{aligned}
$$

LEMMA 8.2 For any $\eta(\cdot, t), \chi(\cdot, t) \in H^{1}(\Omega(t))$, we have

$$
\begin{aligned}
& \frac{\mathrm{d}}{\mathrm{d} t} m(\eta, \chi)=m\left(\partial^{\bullet} \eta, \chi\right)+m\left(\eta, \partial^{\bullet} \chi\right)+q(\eta, \chi), \\
& \frac{\mathrm{d}}{\mathrm{d} t} m(\eta, \chi)=m\left(\partial_{\hat{v}_{h}} \eta, \chi\right)+m\left(\eta, \partial_{\hat{v}_{h}} \chi\right)+\widehat{q}_{h}(\eta, \chi) .
\end{aligned}
$$

On the discrete domain, for $\eta_{h}(\cdot, t), \chi_{h}(\cdot, t) \in S_{h}\left(\Omega_{h}\left(\mathbf{x}^{*}(t)\right)\right)$, we have

$$
\frac{\mathrm{d}}{\mathrm{d} t} m_{h}^{*}\left(\eta_{h}, \chi_{h}\right)=m_{h}^{*}\left(\partial_{v_{h}^{*}}^{*} \eta_{h}, \chi_{h}\right)+m_{h}^{*}\left(\eta_{h}, \partial_{v_{h}^{*}}^{\bullet} \chi_{h}\right)+q_{h}^{*}\left(\eta_{h}, \chi_{h}\right)
$$

Proof. Follows directly from the Leibniz formula (see (Elliott \& Ranner, 2017, Lemma 7.12)).

We are now in position to formulate and prove the required defect estimates.

LEMmA 8.3 Let the domain $\Omega(t)$ and the exact solution $(u, v, X)$ be sufficiently smooth. Then there is a constant $c>0$ and an $h_{0}>0$, such that for all $h \leqslant h_{0}$ and all $t \in[0, T]$, the defects $\mathbf{d}_{\mathbf{v}} \Omega$ and $\mathbf{d}_{\mathbf{u}}$ are bounded by

$$
\begin{aligned}
\left\|\mathbf{d}_{\mathbf{v} \Omega}\right\|_{\star, \mathbf{x}^{*}} & \leqslant c h^{k}, \\
\left\|\mathbf{d}_{\mathbf{u}}\right\|_{\star, \mathbf{x}^{*}} & \leqslant c h^{k} .
\end{aligned}
$$

Proof. We start with estimating $\mathbf{d}_{\mathbf{u}}$. The defect equation (7.2) is equivalent to

$$
\begin{aligned}
m_{h}^{*}\left(d_{u}, \varphi_{h}\right) & =\frac{\mathrm{d}}{\mathrm{d} t} m_{h}^{*}\left(\widetilde{I}_{h} u, \varphi_{h}\right)+a_{h}^{*}\left(\widetilde{I}_{h} u, \varphi_{h}\right)-m_{h}^{*}\left(f, \varphi_{h}\right) \\
& =m_{h}^{*}\left(\partial_{v_{h}^{*}}^{\bullet} \widetilde{I}_{h} u, \varphi_{h}\right)+q_{h}^{*}\left(\widetilde{I}_{h} u, \varphi_{h}\right)+a_{h}^{*}\left(\widetilde{I}_{h} u, \varphi_{h}\right)-m_{h}^{*}\left(f, \varphi_{h}\right)
\end{aligned}
$$

for all $\varphi_{h} \in S_{h}\left(\mathbf{x}^{*}\right)$. The exact solution $u$ satisfies, using Lemma 8.2 and Lemma 8.1,

$$
\begin{aligned}
0 & =\frac{\mathrm{d}}{\mathrm{d} t} m\left(u, \varphi_{h}^{\ell}\right)+a\left(u, \varphi_{h}^{\ell}\right)-m\left(f, \varphi_{h}^{\ell}\right) \\
& =m\left(\partial_{\hat{v}_{h}} u, \varphi_{h}^{\ell}\right)+\widehat{q}_{h}\left(u, \varphi_{h}^{\ell}\right)+a\left(u, \varphi_{h}^{\ell}\right)-m\left(f, \varphi_{h}^{\ell}\right) .
\end{aligned}
$$

Subtracting both terms yields

$$
\begin{aligned}
m_{h}^{*}\left(d_{u}, \varphi_{h}\right) & =\left(m_{h}^{*}\left(\partial_{v_{h}^{*}}^{\bullet} \widetilde{I}_{h} u, \varphi_{h}\right)-m\left(\partial_{\hat{v}_{h}}^{\bullet} u, \varphi_{h}^{\ell}\right)\right)+\left(q_{h}^{*}\left(\widetilde{I}_{h} u, \varphi_{h}\right)-\widehat{q}_{h}\left(u, \varphi_{h}^{\ell}\right)\right) \\
& +\left(a_{h}^{*}\left(\widetilde{I}_{h} u, \varphi_{h}\right)-a\left(u, \varphi_{h}^{\ell}\right)\right)-\left(\left(m_{h}^{*}\left(f, \varphi_{h}\right)-m\left(f, \varphi_{h}^{\ell}\right)\right)\right) .
\end{aligned}
$$


We will estimate the four differences separately.

(i) For the first difference, we use $\partial_{\hat{v}_{h}}^{\bullet} I_{h} u=I_{h} \partial_{\hat{v}_{h}}^{\bullet} u$ :

$$
\left|m_{h}^{*}\left(\partial_{v_{h}^{*}}^{\bullet} \widetilde{I}_{h} u, \varphi_{h}\right)-m\left(\partial_{\hat{v}_{h}}^{\bullet} u, \varphi_{h}^{\ell}\right)\right| \leqslant\left|m_{h}^{*}\left(\partial_{v_{h}^{*}}^{\bullet} \widetilde{I}_{h} u, \varphi_{h}\right)-m\left(\partial_{\widehat{v}_{h}}^{\bullet} I_{h} u, \varphi_{h}^{\ell}\right)\right|+\left|m\left(I_{h} \partial_{\hat{v}_{h}}^{\bullet} u-\partial_{\widehat{v}_{h}}^{\bullet} u, \varphi_{h}^{\ell}\right)\right| .
$$

For the first term, note that $\left(\partial_{v_{h}^{*}}^{\bullet} \widetilde{I}_{h} u\right)^{\ell}=\partial_{\widehat{v}_{h}}^{\bullet} I_{h} u$, so Lemma 5.6 yields

$$
\left|m_{h}^{*}\left(\partial_{v_{h}^{*}}^{\bullet} \widetilde{I}_{h} u, \varphi_{h}\right)-m\left(\partial_{\widehat{v}_{h}}^{\bullet} I_{h} u, \varphi_{h}^{\ell}\right)\right| \leqslant c h^{k}\left\|\partial_{\widehat{v}_{h}}^{\bullet} I_{h} u\right\|_{L^{2}(\Omega)}\left\|\varphi_{h}^{\ell}\right\|_{L^{2}(\Omega)} .
$$

Now we bound

$$
\left\|\partial_{\hat{v}_{h}}^{\bullet} I_{h} u\right\|_{L^{2}(\Omega)}=\left\|I_{h} \partial_{\hat{v}_{h}}^{\bullet} u-\partial_{\hat{v}_{h}}^{\bullet} u+\partial_{\hat{v}_{h}}^{\bullet} u\right\|_{L^{2}(\Omega)} \leqslant\left(c h^{k}+1\right)\left\|\partial_{\hat{v}_{h}}^{\bullet} u-\partial^{\bullet} u+\partial^{\bullet} u\right\|_{L^{2}(\Omega)} \leqslant c
$$

where we have used that $\left\|\partial_{\hat{v}_{h}}^{\bullet} u-\partial^{\bullet} u\right\|_{L^{2}(\Omega)} \leqslant c h^{k+1}$ (see (Elliott \& Ranner, 2017, Lemma 7.14)) and the regularity assumption on $u$. Similarly

$$
\left|m\left(I_{h} \partial_{\hat{v}_{h}}^{\bullet} u-\partial_{\hat{v}_{h}}^{\bullet} u, \varphi_{h}^{\ell}\right)\right| \leqslant\left\|I_{h} \partial_{\hat{v}_{h}}^{\bullet} u-\left.\partial_{\hat{v}_{h}}^{\bullet} u\right|_{L^{2}(\Omega)}\right\| \varphi_{h}^{\ell}\left\|_{L^{2}(\Omega)} \leqslant c h^{k}\right\| \partial_{\hat{v}_{h}}^{\bullet} u\|\| \varphi_{h}^{\ell}\left\|_{L^{2}(\Omega)} \leqslant c h^{k}\right\| \varphi_{h}^{\ell} \|_{L^{2}(\Omega)} .
$$

Altogether, we have for the first difference of (8.1)

$$
\left|m_{h}^{*}\left(\partial_{v_{h}^{*}}^{\bullet} \widetilde{I}_{h} u, \varphi_{h}\right)-m\left(\partial_{\widehat{v}_{h}}^{\bullet} u, \varphi_{h}^{\ell}\right)\right| \leqslant c h^{k}\left\|\varphi_{h}^{\ell}\right\|_{L^{2}(\Omega)} .
$$

(ii) In a similar way:

$$
\left|q_{h}^{*}\left(\widetilde{I}_{h} u, \varphi_{h}\right)-\widehat{q}_{h}\left(u, \varphi_{h}^{\ell}\right)\right| \leqslant\left|q_{h}^{*}\left(\widetilde{I}_{h} u, \varphi_{h}\right)-\widehat{q}_{h}\left(I_{h} u, \varphi_{h}^{\ell}\right)\right|+\left|\widehat{q}_{h}\left(I_{h} u-u, \varphi_{h}^{\ell}\right)\right| .
$$

For the first term, we use (Elliott \& Ranner, 2017, Lemma 7.15):

$$
\left|q_{h}^{*}\left(\widetilde{I}_{h} u, \varphi_{h}\right)-\widehat{q}_{h}\left(I_{h} u, \varphi_{h}^{\ell}\right)\right| \leqslant c h^{k+1}\left\|I_{h} u\right\|_{L^{2}(\Omega)}\left\|\varphi_{h}^{\ell}\right\| \leqslant c h^{k+1}\left\|\varphi_{h}^{\ell}\right\|_{L^{2}(\Omega)} .
$$

For the second term, we use an $L^{2}-L^{2}-L^{\infty}$ estimate and (Elliott \& Ranner, 2017, Lemma 7.14) to bound $\left\|\nabla \widehat{v}_{h}\right\|_{L^{\infty}(\Omega)}$ :

$$
\begin{aligned}
\left|\widehat{q}_{h}\left(I_{h} u-u, \varphi_{h}^{\ell}\right)\right| & \leqslant\left\|I_{h} u-u\right\|_{L^{2}(\Omega)}\left\|\varphi_{h}^{\ell}\right\|_{L^{2}(\Omega)}\left\|\nabla \cdot \widehat{v}_{h}\right\|_{L^{\infty}(\Omega)} \\
& \leqslant c h^{k+1}\left\|\varphi_{h}^{\ell}\right\|_{L^{2}(\Omega)}\left\|\nabla \widehat{v}_{h}\right\|_{L^{\infty}(\Omega)} \leqslant c h^{k+1}\left\|\varphi_{h}^{\ell}\right\|_{L^{2}(\Omega)} .
\end{aligned}
$$

(iii) The third term of (8.1) is estimated similarly:

$$
\begin{aligned}
\left|a_{h}^{*}\left(\widetilde{I}_{h} u, \varphi_{h}\right)-a\left(u, \varphi_{h}^{\ell}\right)\right| & \leqslant\left|a_{h}^{*}\left(\widetilde{I}_{h} u, \varphi_{h}\right)-a\left(I_{h} u, \varphi_{h}^{\ell}\right)\right|+\left|a\left(I_{h} u-u, \varphi_{h}^{\ell}\right)\right| \\
& \leqslant c h^{k}\left\|\nabla I_{h} u\right\|_{L^{2}(\Omega)}\left\|\nabla \varphi_{h}^{\ell}\right\|_{L^{2}(\Omega)}+\left\|\nabla\left(I_{h} u-u\right)\right\|_{L^{2}(\Omega)}\left\|\nabla \varphi_{h}^{\ell}\right\|_{L^{2}(\Omega)} \\
& \leqslant c h^{k}\left\|\varphi_{h}^{\ell}\right\|_{H^{1}(\Omega)} .
\end{aligned}
$$


(iv) For the last term of (8.1), we immediately have

$$
\left|m_{h}^{*}\left(f, \varphi_{h}\right)-m\left(f, \varphi_{h}^{\ell}\right)\right| \leqslant c h^{k}\|f\|_{L^{2}(\Omega)}\left\|\varphi_{h}^{\ell}\right\|_{L^{2}(\Omega)} .
$$

Putting those four estimates together, using norm equivalence, we obtain

$$
\left\|\mathbf{d}_{\mathbf{u}}\right\|_{\star, \mathbf{x}^{*}}=\left\|d_{u}\right\|_{H_{h}^{-1}\left(\Omega\left(\mathbf{x}^{*}\right)\right)}=\sup _{0 \neq \varphi_{h} \in S_{h}\left(\mathbf{x}^{*}\right)} \frac{m_{h}^{*}\left(d_{u}, \varphi_{h}\right)}{\left\|\varphi_{h}\right\|_{H^{1}\left(\Omega\left(\mathbf{x}^{*}\right)\right)}} \leqslant c h^{k} .
$$

Now we estimate $\mathbf{d}_{\mathbf{v}} \Omega$, which is defined by the defect equation (6.2). We set $\mathbf{d}_{\mathbf{v}}^{\mathrm{T}}=\left(0, \mathbf{d}_{\mathbf{v} \Omega}^{\mathrm{T}}\right)$ and $\mathbf{w}^{\mathrm{T}}=\left(0, \mathbf{w}^{\Omega, \mathrm{T}}\right)$ for $\mathbf{w}^{\Omega} \in \mathbb{R}^{3 N_{\Omega}}$ and test with $\mathbf{w}^{\Omega}$ to obtain with a computation similar to (6.11) (omitting the tensor notation) $\mathbf{w}^{\mathrm{T}} \mathbf{M}\left(\mathbf{x}^{*}\right) \mathbf{d}_{\mathbf{v}}=\mathbf{w}^{\mathrm{T}} \mathbf{A}\left(\mathbf{x}^{*}\right) \mathbf{v}^{*}$ which is equivalent to

$$
\int_{\Omega_{h}\left(\mathbf{x}^{*}\right)} \varphi_{h} \cdot d_{h} \mathrm{~d} x=\int_{\Omega_{h}\left(\mathbf{x}^{*}\right)} \nabla \varphi_{h} \cdot \nabla v_{h}^{*} \mathrm{~d} x=a_{h}\left(\varphi_{h}, \widetilde{I}_{h} v\right)-a\left(\varphi_{h}^{\ell}, I_{h} v\right)+a\left(\varphi_{h}^{\ell}, I_{h} v\right)
$$

for all $\varphi_{h} \in S_{0, h}\left(\mathbf{x}^{*}\right)$. We will estimate the first difference and the second term of (8.2) separately, starting with the second term. Since $\varphi_{h} \in S_{0, h}\left(\mathbf{x}^{*}\right)$, we have $\varphi_{h}^{\ell} \in H_{0}^{1}(\Omega(t))$ and thus $a\left(\varphi_{h}^{\ell}, v\right)=0$. With Proposition 3.2, we obtain

$$
a\left(\varphi_{h}^{\ell}, I_{h} v\right)=a\left(\varphi_{h}^{\ell}, I_{h} v-v\right) \leqslant\left\|\nabla \varphi_{h}^{\ell}\right\|_{L^{2}(\Omega)}\left\|\nabla\left(I_{h} v-v\right)\right\|_{L^{2}(\Omega)} \leqslant c h^{k}\left\|\nabla \varphi_{h}^{\ell}\right\|_{L^{2}(\Omega)}\|v\|_{H^{k+1}(\Omega)} .
$$

The first difference in (8.2) is estimated analogously to (iii) in the first part of this proof and yields

$$
\left|a_{h}\left(\varphi_{h}, \widetilde{I}_{h} v\right)-a\left(\varphi_{h}^{\ell}, I_{h} v\right)\right| \leqslant c h^{k}\left\|\nabla \varphi_{h}^{\ell}\right\|_{L^{2}(\Omega)}
$$

for $h \leqslant h_{0}$ sufficiently small using the regularity assumption.

Putting these estimates together yields with Lemma 5.5:

$$
\left\|\mathbf{d}_{\mathbf{v} \Omega}\right\|_{\star, \mathbf{x}^{*}}=\sup _{0 \neq \mathbf{w}^{\Omega} \in \mathbb{R}^{3 N_{\Omega}}} \frac{\mathbf{d}_{\mathbf{v}^{\Omega}}^{\mathrm{T}} \mathbf{M}_{22}(\mathbf{x} *) \mathbf{w}^{\Omega}}{\left\|\mathbf{w}^{\Omega}\right\|_{\mathbf{A}_{22}\left(\mathbf{x}^{*}\right)}} \leqslant c h^{k} .
$$

\section{Proof of Theorem 4.1}

We prove the first error bound. The remaining ones are shown analogously. The error is decomposed using interpolation and lift:

$$
u_{h}^{L}-u=\left(\widehat{u}_{h}-\widetilde{I}_{h} u\right)^{\ell}+\left(I_{h} u-u\right)
$$

The right term can be bounded by $c h^{k}$ in the $H^{1}$-norm using an interpolation estimate. For the first term we obtain, using norm equivalence, Lemma 6.1 and Lemma 8.3

$$
\begin{aligned}
\left\|\left(\widehat{u}_{h}-\widetilde{I}_{h} u\right)^{\ell}\right\|_{L^{2}(\Omega(t))} & \leqslant c\left\|\widehat{u}_{h}-\widetilde{I}_{h} u\right\|_{L^{2}\left(\Omega_{h}\left(\mathbf{x}^{*}(t)\right)\right)}=c\left\|\mathbf{e}_{\mathbf{u}}\right\|_{\mathbf{M}\left(\mathbf{x}^{*}\right)} \\
& \leqslant c \int_{0}^{t}\left\|\mathbf{d}_{\mathbf{u}}(s)\right\|_{\star, \mathbf{x}^{*}}^{2}+\left\|\mathbf{d}_{\mathbf{v}}(s)\right\|_{\star, \mathbf{x}^{*}}^{2} \mathrm{~d} s \leqslant c h^{k} .
\end{aligned}
$$

Analogously

$$
\left\|\nabla\left(\widehat{u}_{h}-\widetilde{I}_{h} u\right)^{\ell}\right\|_{L^{2}(\Omega(t))} \leqslant c\left\|\nabla\left(\widehat{u}_{h}-\widetilde{I}_{h} u\right)\right\|_{L^{2}\left(\Omega_{h}\left(\mathbf{x}^{*}(t)\right)\right)}=c\left\|\mathbf{e}_{\mathbf{u}}\right\|_{\mathbf{A}\left(\mathbf{x}^{*}\right)} .
$$

Lemma 7.2 and Lemma 8.3 yield the result. The remaining estimates are shown analogously. 


\section{REMARK 9.1 ( $L^{2}$-estimate)}

The convergence rate in $u, v$ and $X$ in the $L^{2}$-norm is expected to be of order $k+1$, which is also reflected in the numerical experiments down below. In order to prove $\mathscr{O}\left(h^{k+1}\right)$-error bounds for the diffusion equation, one could work with the Ritz projection $R_{h} u$ instead of the interpolation $I_{h} u$. In fact, defining a Ritz projection as described in (Elliott \& Ranner, 2017, Section 3.3.2), cf. Dziuk \& Elliott (2013), we are able to prove $\sup _{t \in[0, T]}\left\|\mathbf{d}_{\mathbf{u}}(t)\right\|_{\star, \mathbf{x}^{*}(t)} \leqslant c h^{k+1}$. This yields the error bound

$$
\left\|\mathbf{e}_{\mathbf{u}}(t)\right\|_{\mathbf{M}\left(\mathbf{x}^{*}\right)}^{2}+\int_{0}^{t}\left\|\mathbf{e}_{\mathbf{u}}(s)\right\|_{\mathbf{A}\left(\mathbf{x}^{*}(s)\right)}^{2} \mathrm{~d} s \leqslant C h^{2 k+2}+c \int_{0}^{t}\left\|\mathbf{d}_{\mathbf{v}}(s)\right\|_{\star, \mathbf{x}^{*}(s)}^{2} \mathrm{~d} s .
$$

It is further possible to define a Ritz map for the Laplace equation for the velocity, taking the inhomogeneous boundary conditions into account. However, taking the Ritz projection instead of the finite element interpolation implies that the corresponding error $\mathbf{e}_{\mathbf{v}}$ does not vanish on the boundary anymore. This induces a different defect $\mathbf{d}_{\mathbf{v}}$ in $v$ and an additional defect $\mathbf{d}_{\mathbf{x}}$ in the equation $\dot{\mathbf{e}}_{\mathbf{x}}=\mathbf{e}_{\mathbf{v}}+\mathbf{d}_{\mathbf{x}}$, where $\mathbf{d}_{\mathbf{x}}$ can be considered as the error between the finite element interpolation $I_{h} v$ and the Ritz projection $R_{h} v$ of $v$. While it is indeed possible to obtain an $\mathscr{O}\left(h^{k+1}\right)$ bound for $\mathbf{d}_{\mathbf{v}}$, this is no longer true for the new defect $\mathbf{d}_{\mathbf{x}}$, which still has to be estimated in the A-norm, see (6.13), yielding only a $h^{k}$ error bound.

\section{Numerical experiments}

In this section we illustrate the theoretical results with various numerical experiments. Fitting the layout of the stability proof, we start with an evolving domain problem in two dimensions without solving a diffusion equation on that domain. The second example is similar to the first one but three-dimensional. In the third example, we show convergence plots for a diffusion equation with non-homogeneous Neumann boundary conditions on a rotating and growing sphere.

All experiments were implemented in MATLAB ${ }^{\circledR} \mathrm{R} 2018 \mathrm{a}$ and performed in reasonable time on an MSI GE63VR notebook with Intel Core i7-7700HQ processor and 16 GB DDR4-RAM.

\subsection{An evolving open domain}

We consider problem (2.5) for $t \in[0,1]$, with $\Omega(0)$ being the unit circle in $\mathbb{R}^{2}$. As exact solution, we choose

$$
v(x, t)=\left(\begin{array}{c}
\exp (-2 t)\left(\exp \left(x_{1}\right) \sin \left(x_{2}\right)-\exp \left(x_{2}\right) \sin \left(x_{1}\right)\right) \\
2 \exp (-5 t)\left(x_{1}^{2}-x_{2}^{2}\right)
\end{array}\right),
$$

which satisfies $-\Delta v=0$. Exemplary triangulations of $\Omega\left(t_{j}\right)$ for $t_{j}=j / 5, j=0, \ldots, 5$ are shown in Figures 1 and 2 .

We apply a second order isoparametric finite element method. For time discretization, we use a linearly implicit 4-step BDF method with time step size $\tau=8 \cdot 10^{-3}$, such that the time discretization error is negligible. To compute a reference solution, we use the fact that the above $v$ satisfies $-\Delta v(\cdot, t)=$ 0 , and solve the position ODEs

$$
\frac{\mathrm{d}}{\mathrm{d} t} x_{j}(t)=v\left(x_{j}(t), t\right), \quad x_{j}(0)=x_{j}^{0},
$$

in all nodes $x_{j}^{0}, j=1, \ldots, N$, of the initial triangulation with a RK4 method and time step size $\tau=$ $2 \cdot 10^{-4}$. Since stiffness is no issue in the position ODEs, an explicit high-order time discretization scheme is sufficient to compute a reference solution. 
We record the position error

$$
\begin{aligned}
&\left\|\operatorname{err}_{\mathbf{x}}\right\|_{L^{\infty}\left(L^{2}\right)}:=\sup _{n: n \tau \leqslant 1}\left\|\left(x_{h}^{n}\right)^{L}-\operatorname{id}_{\Omega\left(t_{n}\right)}\right\|_{L^{2}\left(\Omega\left(t_{n}\right)\right)^{2}}, \\
&\left\|\operatorname{err}_{\mathbf{x}}\right\|_{L^{\infty}\left(H^{1}\right)}:=\sup _{n: n \tau \leqslant 1}\left\|\nabla\left(\left(x_{h}^{n}\right)^{L}-\operatorname{id}_{\Omega\left(t_{n}\right)}\right)\right\|_{L^{2}\left(\Omega\left(t_{n}\right)\right)^{2}} .
\end{aligned}
$$

and the velocity error err $_{\mathbf{v}}$ in the same norms for different choices of $h$. Figure 3 shows the results. The error in $H^{1}$-norm converges with the expected order, whereas the convergence rate of the $L^{2}$-norm error is one order higher. This is not covered by the theory of this paper and left to possible future works.

REMARK 10.1 (Linear finite elements) We solved the same problem with linear finite elements. Although not covered by the theory of this paper, we observe the expected $\mathscr{O}\left(h^{2}\right)$-convergence in $L^{2}$-norm and $\mathscr{O}(h)$-convergence in $H^{1}$-norm.

\subsection{An evolving 3d domain}

This example is similar to the previous one, but in three dimensions. We consider (2.5) for $t \in[0,0.1]$, with $\Omega(0)$ being the unit ball in $\mathbb{R}^{3}$. As exact solution, we choose

$$
v(x, t)=\left(\begin{array}{c}
\sin (-t / 10)\left(x_{1}^{2}-2 x_{2}^{2}+x_{3}^{2}\right) \\
\sin (-t / 10)\left(\exp \left(x_{2}\right) \sin \left(x_{3}\right)-\exp \left(x_{3}\right) \sin \left(x_{2}\right)\right) \\
\exp (-5 t)\left(x_{1}^{2}-x_{3}^{2}\right)
\end{array}\right)
$$

which satisfies $-\Delta v=0$. We use isoparametric finite elements of second order. For the time discretization and reference solution, we proceed as in the previous example, with $\tau=10^{-3}$ for the BDF method and $\tau=10^{-6}$ for the reference solution. We record the $L^{\infty}\left(L^{2}\right)$ - and $L^{\infty}\left(H^{1}\right)$-norm of the position and velocity error. The results are shown in Figure 4.
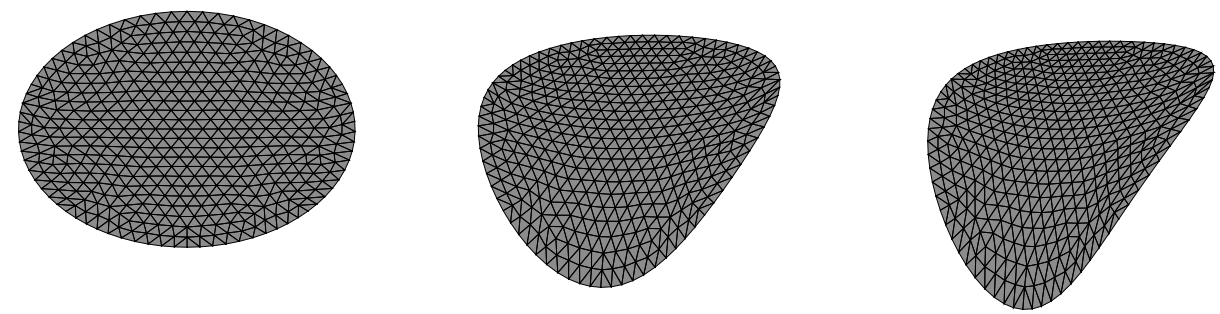

FIG. 1. Triangulation of $\Omega(t)$ at $t_{0}=0$ (left), $t_{1}=0.2$ (center) and $t_{2}=0.4$ (right). 

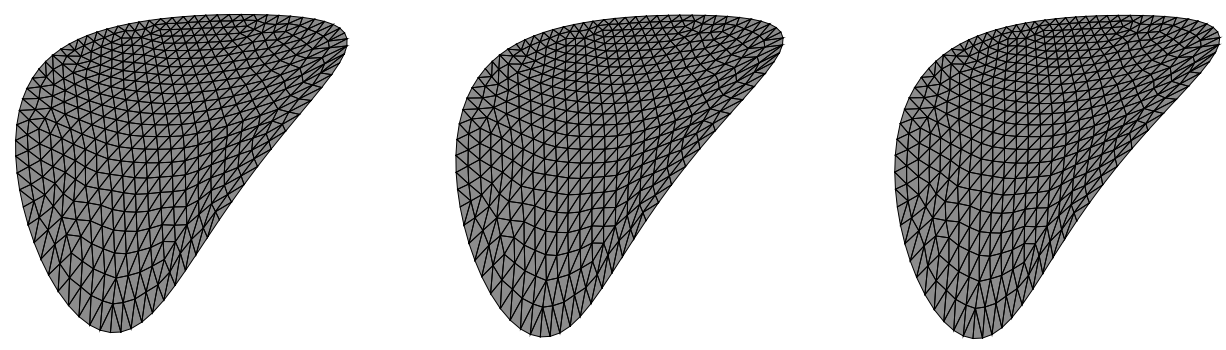

FIG. 2. Triangulation of $\Omega(t)$ at $t_{3}=0.6$ (left), $t_{4}=0.8$ (center) and $t_{5}=1.0$ (right).
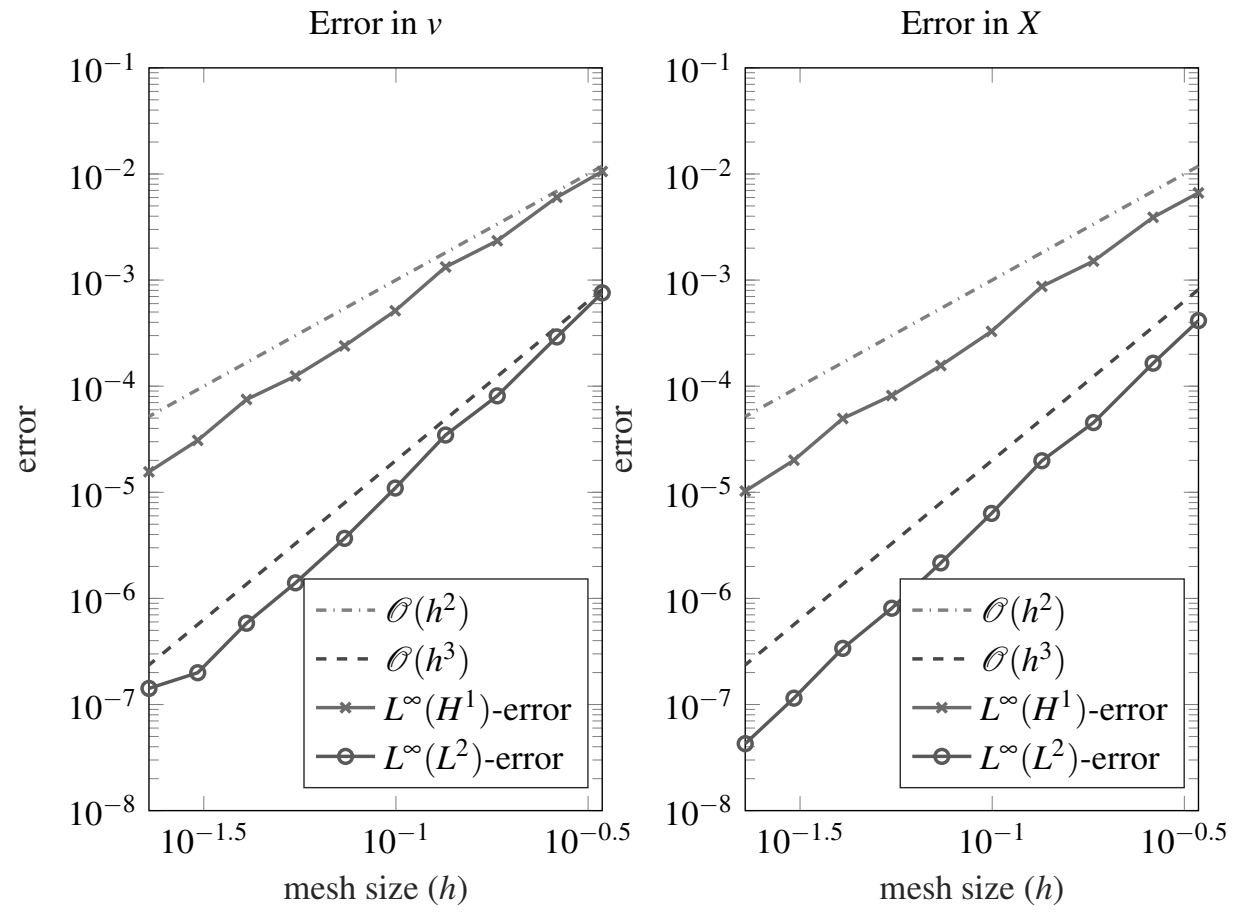

FIG. 3. Convergence rate of the evolving quadratic finite element discretization of Example 10.1.

\subsection{Diffusion equation}

In this example, we consider the diffusion equation (2.4), where the velocity again satisfies (2.5). As exact solution, we choose $\beta=1$ and

$$
\begin{aligned}
& u(x, y, t)=e^{-t}\left(x^{2}+y^{2}\right)\left(x^{2}-y^{2}\right) \\
& v(x, y, t)=\left(1-\frac{1}{r(t)}\right)\left(\begin{array}{l}
x \\
y
\end{array}\right)+\left(\begin{array}{c}
-y \\
x
\end{array}\right), \quad \text { where } \quad r(t)=\frac{2}{1+e^{-t}}
\end{aligned}
$$



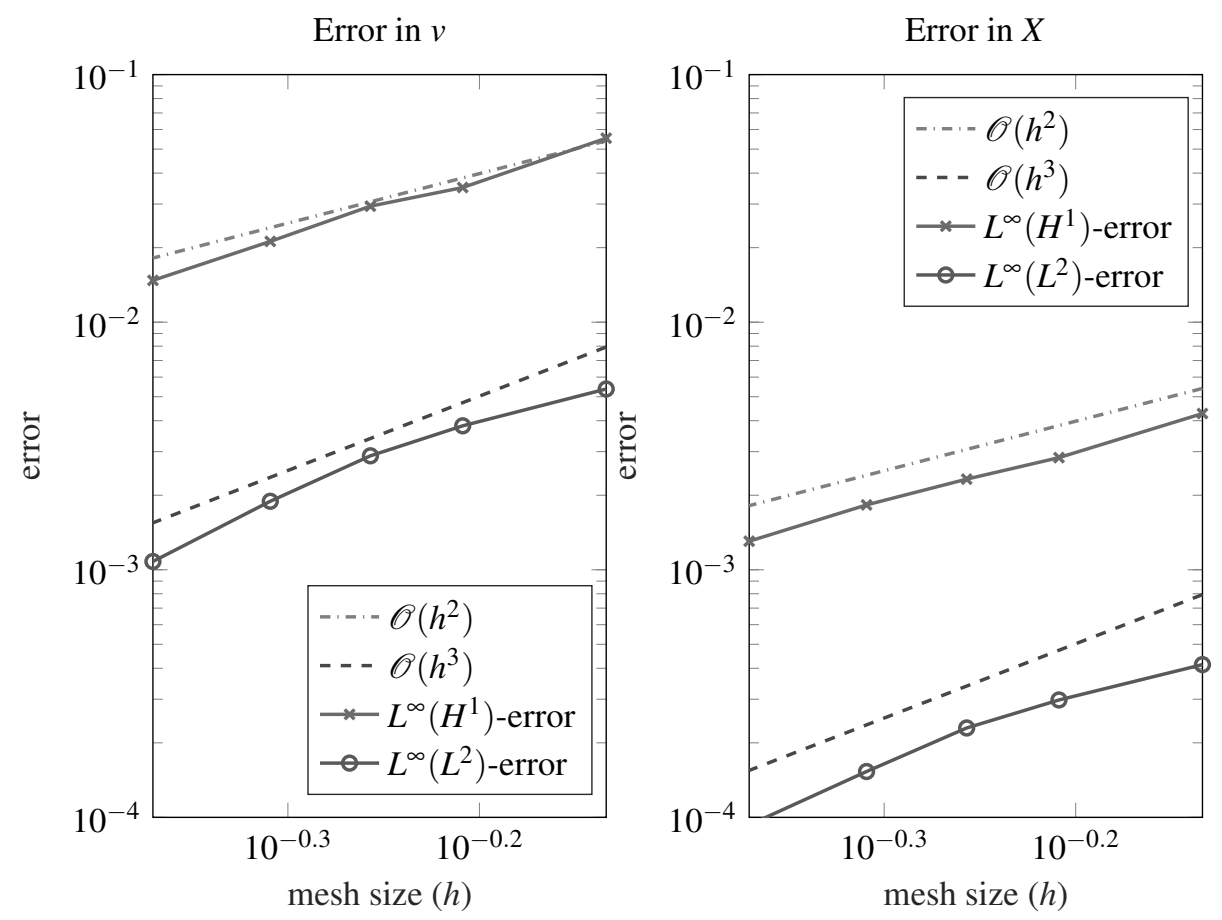

FIG. 4. Convergence rate of the evolving quadratic finite element discretization of Example 10.2.

The velocity $v$ describes a growing ball which in addition is rotating anti-clockwise (cf. (Kovács et al., 2017, Example 11.1)), $r(t)$ is the radius of the ball at $t \in[0, T]$. We compute the right-hand side functions $f$ and $g$ of (2.4) and apply second order isoparametric finite elements in space and a linearly implicit 4 step BDF method with time step-size $\tau=10^{-3}$ in time.

Note that $v$ is linear in $x$ and $y$, so the solution to $-\Delta v=0$ is computed exactly by the finite element method. This is reflected in the convergence plot in $v$, which shows a purely temporal convergence and is thus not shown here. We record the error

$$
\begin{aligned}
& \left\|\operatorname{err}_{\mathbf{u}}\right\|_{L^{\infty}\left(L^{2}\right)}:=\sup _{n: t_{n} \leqslant 1}\left\|\left(u_{h}^{n}\right)^{L}-u\left(\cdot, t_{n}\right)\right\|_{L^{2}\left(\Omega\left(t_{n}\right)\right)}, \\
& \left\|\operatorname{err}_{\mathbf{u}}\right\|_{L^{2}\left(H^{1}\right)}:=\left(\tau \sum_{n: t_{n} \leqslant 1}\left\|\left(u_{h}^{n}\right)^{L}-u\left(\cdot, t_{n}\right)\right\|_{H^{1}\left(\Omega\left(t_{n}\right)\right)}^{2}\right)^{\frac{1}{2}},
\end{aligned}
$$

where $\tau$ denotes the time step size and $t_{n}=n \tau$ the $n$-th time step. Figure 5 shows the results. As expected, the error in the $L^{2}\left(H^{1}\right)$-norm converges with the expected order, whereas the $L^{2}$-norm convergence rate is one order higher. 


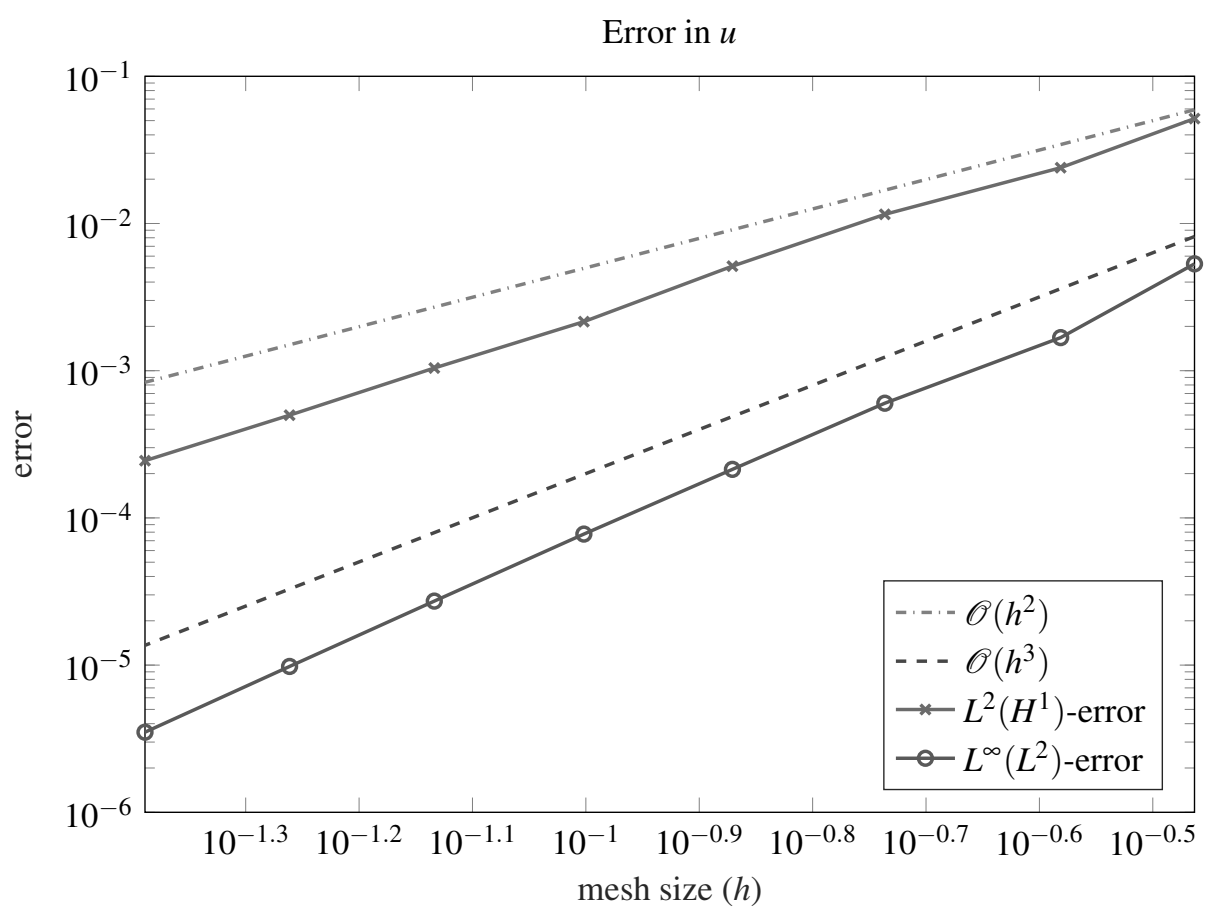

FIG. 5. Convergence rate in $u$ of the evolving quadratic finite element discretization of Example 10.3.

\section{Acknowledgments}

The author is very grateful to Christian Lubich and Balázs Kovács for stimulating discussions on the topic and their help during the work on this manuscript. We thank the anonymous referees for helpful and constructive comments.

\section{References}

Badia, S., \& Codina, R. 2006. Analysis of a stabilized finite element approximation of the transient convection-diffusion equation using an ALE framework. SIAM Journal on Numerical Analysis, 44(5), 2159-2197.

Bernardi, C. 1989. Optimal finite-element interpolation on curved domains. SIAM Journal on Numerical Analysis, 26(5), 1212-1240.

Boffi, D., \& Gastaldi, L. 2004. Stability and geometric conservation laws for ALE formulations. Computer Methods in Applied Mechanics and Engineering, 193(42-44), 4717-4739.

Brenner, S., \& Scott, R. 2007. The mathematical theory of finite element methods. Vol. 15. Springer Science \& Business Media. 
Ciarlet, P.G., \& Raviart, P.A. 1972. Interpolation theory over curved elements, with applications to finite element methods. Computer Methods in Applied Mechanics and Engineering, 1(2), 217-249.

Dziuk, G., \& Elliott, C.M. 2007a. Finite elements on evolving surfaces. IMA Journal of Numerical Analysis, 27(2), 262-292.

Dziuk, G., \& Elliott, C.M. 2007b. Surface finite elements for parabolic equations. Journal of Computational Mathematics, 385-407.

Dziuk, G., \& Elliott, C.M. 2013. $L^{2}$-estimates for the evolving surface finite element method. Mathematics of Computation, 82(281), 1-24.

Dziuk, G., Lubich, C., \& Mansour, D. 2012. Runge-Kutta time discretization of parabolic differential equations on evolving surfaces. IMA Journal of Numerical Analysis, 32(2), 394-416.

Dziuk, G., Kröner, D., \& Müller, T. 2013. Scalar conservation laws on moving hypersurfaces. arXiv preprint arXiv:1307.1056.

Elliott, C.M., \& Ranner, T. 2013. Finite element analysis for a coupled bulk-surface partial differential equation. IMA Journal of Numerical Analysis, 33(2), 377-402.

Elliott, C.M., \& Ranner, T. 2017. A unified theory for continuous in time evolving finite element space approximations to partial differential equations in evolving domains. arXiv preprint arXiv:1703.04679.

Eyles, J., King, J. F., \& Styles, V. 2019. A tractable mathematical model for tissue growth. arXiv preprint arXiv:1907.06590.

Formaggia, L., \& Nobile, F. 1999. A stability analysis for the arbitrary Lagrangian Eulerian formulation with finite elements. East-West Journal of Numerical Mathematics, 7(ARTICLE), 105-132.

Formaggia, L., \& Nobile, F. 2004. Stability analysis of second-order time accurate schemes for ALEFEM. Computer Methods in Applied Mechanics and Engineering, 193(39-41), 4097-4116.

Gastaldi, L. 2001. A priori error estimates for the arbitrary Lagrangian Eulerian formulation with finite elements. Journal of Numerical Mathematics, 9(2), 123-156.

Kovács, B. 2017. High-order evolving surface finite element method for parabolic problems on evolving surfaces. IMA Journal of Numerical Analysis, 38(1), 430-459.

Kovács, B., \& Lubich, C. 2018. Linearly implicit full discretization of surface evolution. Numerische Mathematik, 140(1), 121-152.

Kovács, B., Li, B., Lubich, C., \& Power Guerra, C.A. 2017. Convergence of finite elements on a solution-driven evolving surface. Numerische Mathematik, 137, 643-689.

Kovács, B., Li, B., \& Lubich, C. 2019a. A convergent algorithm for forced mean curvature flow driven by diffusion on the surface. arXiv preprint arXiv:1912.05924, to appear in Interfaces and Free Boundaries.

Kovács, B., Li, B., \& Lubich, C. 2019b. A convergent evolving finite element algorithm for mean curvature flow of closed surfaces. Numerische Mathematik, 143(4), 797-853. 